\title{
Dynamic Analysis of Cable-Stayed Bridges Affected by Accidental Failure Mechanisms under Moving Loads
}

\author{
Fabrizio Greco, Paolo Lonetti, and Arturo Pascuzzo \\ Department of Structural Engineering, University of Calabria, P. Bucci Road, Cubo39-B, Rende, 87030 Cosenza, Italy \\ Correspondence should be addressed to Paolo Lonetti; lonetti@unical.it
}

Received 21 August 2012; Accepted 14 November 2012

Academic Editor: Xiaojun Wang

Copyright ( 2013 Fabrizio Greco et al. This is an open access article distributed under the Creative Commons Attribution License, which permits unrestricted use, distribution, and reproduction in any medium, provided the original work is properly cited.

\begin{abstract}
The dynamic behavior of cable-stayed bridges subjected to moving loads and affected by an accidental failure in the cable suspension system is investigated. The main aim of the paper is to quantify, numerically, the dynamic amplification factors of typical kinematic and stress design variables, by means of a parametric study developed in terms of the structural characteristics of the bridge components. The bridge formulation is developed by using a geometric nonlinear formulation, in which the effects of local vibrations of the stays and of large displacements in the girder and the pylons are taken into account. Explicit time dependent damage laws, reproducing the failure mechanism in the cable system, are considered to investigate the influence of the failure mode characteristics on the dynamic bridge behavior. The analysis focuses attention on the influence of the inertial characteristics of the moving loads, by accounting coupling effects arising from the interaction between girder and moving system. Sensitivity analyses of typical design bridge variables are proposed. In particular, the effects produced by the moving system characteristics, the tower typologies, and the failure mode characteristics involved in the cable system are investigated by means of comparisons between damaged and undamaged bridge configurations.
\end{abstract}

\section{Introduction}

Cable supported bridges are frequently employed in the context of long spans, leading to slender structures, in which, typically, the dead loads are comparable with those involved in the live load configuration. As a consequence, the external loads are able to produce high amplifications of the main bridge kinematic and stress design parameters, leading to nonstandard excitation modes and unexpected damage mechanisms in the structural components of the bridge. In order to simulate the actual behavior of the bridge, the structural modeling should be able to reproduce the source of nonlinearities in the cable system, in the girder or in the pylons due to cable sag or beam-column large deformation effects, respectively. Moreover, additional complexities arise in the prediction of the bridge behavior in the presence of damage mechanisms, which strongly reduce the structural integrity of the bridge. As a matter of fact, cable-supported structures are, typically, affected by degradation effects such as corrosion, abrasion, and fatigue, which may cause a reduction of the stiffness properties or, in extreme cases, the complete failure of a single or multiple cable elements. In the literature, many investigations have been developed to analyze the influence of the moving loads on the dynamic behavior of cablesupported bridges, mainly, for undamaged bridge structures. Actually, many models are devoted to predicting dynamic bridge behavior by using refined structural schematizations as well as accurate descriptions of the moving loads [1-4]. In this framework, the bridge behavior is analyzed by means of analytical continuum approaches or finite element models, in which, the behavior of the cable suspension system is typically described by means of linear equations expressed in terms of tangent or secant Dischinger elastic moduli [5-8]. This assumption is frequently supported by experimental evidence for static analyses $[9,10]$. However, in order to reproduce the dynamic behavior correctly, especially when the bridge is subjected to extreme loading conditions, local vibration effects of the cable elements should be, properly, taken into account $[2,11]$. Additional complexities in the prediction of the dynamic behavior of long span bridges arise from the description of the interaction behavior between moving loads and bridge vibrations. At this aim, many papers have been 
developed to analyze the influence of the external mass and its motion on the bridge behavior, introducing an accurate description of the inertial forces between bridge deformation and moving load kinematic $[7,8,12]$. However, a brief literature review denotes that the behavior of cable-stayed bridges is mainly analyzed for undamaged structures, whereas the influence of cable failure mechanisms produced by loss of stiffness due to cable degradation or due to an accidental failure is rarely analyzed. Typically, many papers are devoted to investigating the effects on the dynamic behavior of a single cable element of a localized or a distributed time independent damage mechanisms [13], without entering in detail on time dependence characteristics of the failure mode or the coupling behavior between damaged and undamaged elements of the cable system. The results proposed in this framework show how damage phenomena induce tension loss and sag augmentation of the static undamaged cable profile.

Since cable elements are mostly utilized in supported bridges, studies developed on single cables have been generalized to evaluating the influence of damage phenomena on realistic cable structures. In particular, damage behavior of cable-stayed beam has been analyzed by means of numerical approaches, in which the effects of diffused inelastic damage concerning the intensity and location characteristics have been investigated [14]. Such results show how the damage effects produce relevant modifications with respect to the undamaged configuration of the natural frequencies and mode shapes of the structure. Moreover, further investigation has been developed in the framework of cable-stayed bridge.

It is worth noting that from a design point of view, existing codes on cable-stayed bridges, that is, PTI [15] and SETRA [16], in order to identify the amplification effects provided by the failure mechanism in the cable system, recommend to amplify the results obtained in the framework of quasistatic analyses by using fictitious amplification factors suggested in the range between 1.5 and 2.0. In particular, the codes identify the dynamic characteristics of the failure mode of a generic element of the cable system, introducing a static loading configuration, in which the cable failure is reproduced by means of compression forces to simulate cable release. The stress distribution arising from such a loading scheme is combined with the effects of other existing loading schemes by means of proper factored loading combinations. However, recent papers have demonstrated that such a simplified approach becomes unsafe in many cases, leading to dynamic amplification factors higher than those suggested by existing recommendations [17-19]. In particular, some parametric studies have been developed for bridge typologies subjected to accidental cable failure by using a numerical approach based on classical standard linear dynamic framework [18, 19]. Such analyses denote that the results obtained by using such code prescriptions are affected by high underestimations in the prediction of typical design bridge variables related to the girder and pylons. However, in order to correctly reproduce the bridge behavior, additional developments are required to be simulate the effect produced by the nonlinearities and damage mechanisms involved cable system and by the inertial coupling between girder and moving loads deformations. Actually, comprehensive analyses that include such non linear dynamic effects are quite rare and thus further investigations to verify code prescriptions and to quantify the influence on the bridge behavior of the dynamic excitation produced by the failure mode characteristics of the cable system are much required.

In the present paper, the dynamic behavior of cablestayed bridges subjected to cable failure mechanisms is investigated. The numerical study consists on a tridimensional finite element model, in which both A-shaped and $\mathrm{H}$-shaped pylons are investigated. The aim of the paper is to propose a parametric study to investigate the influence on the bridge behavior of the dynamic excitation produced by damage mechanisms in the cable system and the transit of moving loads. Differently from existing papers available in the literature, the proposed modeling reproduces the inertial description of the moving loads by means of a refined schematization of the inertial forces produced by the moving system and girder bridge interaction. Moreover, local vibrations of the cable elements are taken into account by reproducing the nonlinearities involved in the cable-sag effect in the cable system as well as large deformations in the girder and the pylons. Finally, the stay failure mechanism is formulated, consistently with a Continuous Damage Mechanics approach, introducing time dependent damage functions, which control the constitutive behavior of the failure mechanism and thus the inertial characteristics of the loss of cable stiffness. The outline of the paper is as follows: Section 2 presents the general formulation of the cable and the girder elements, the damage description of the cable model, and the evaluation of the initial configuration; the numerical implementation is reported in Section 3; parametric studies in terms of bridge and moving loads characteristics and failure mode typology in the cable system are reported in Section 4.

\section{Bridge Formulation}

In this section the governing equations for the bridge constituents as well as the main assumptions concerning the kinematic modeling are discussed. In particular, the main assumptions concerning the kinematic modeling of the bridge, the damage formulation adopted to simulate cable failure, and the inertial forces to define the moving loads/girder interaction are discussed. Such governing equations represent the basis for the theoretical formulation of the model, whose numerical implementation is presented in Section 3.

\subsection{Cable System Formulation and Initial Configuration under} Dead Loading. The bridge scheme is based on a tridimensional modeling, in which both in plane and out of plane deformation modes are considered. The structural model, reported in Figure 1, is consistent with a fan-shaped and a self-anchored cable-stayed bridge scheme. Moreover, the pylons, analyzed by the present paper, refer to A- or $\mathrm{H}$ shaped typologies. Every single cable of the suspension system is simulated by using a sequence of the $n$ truss 


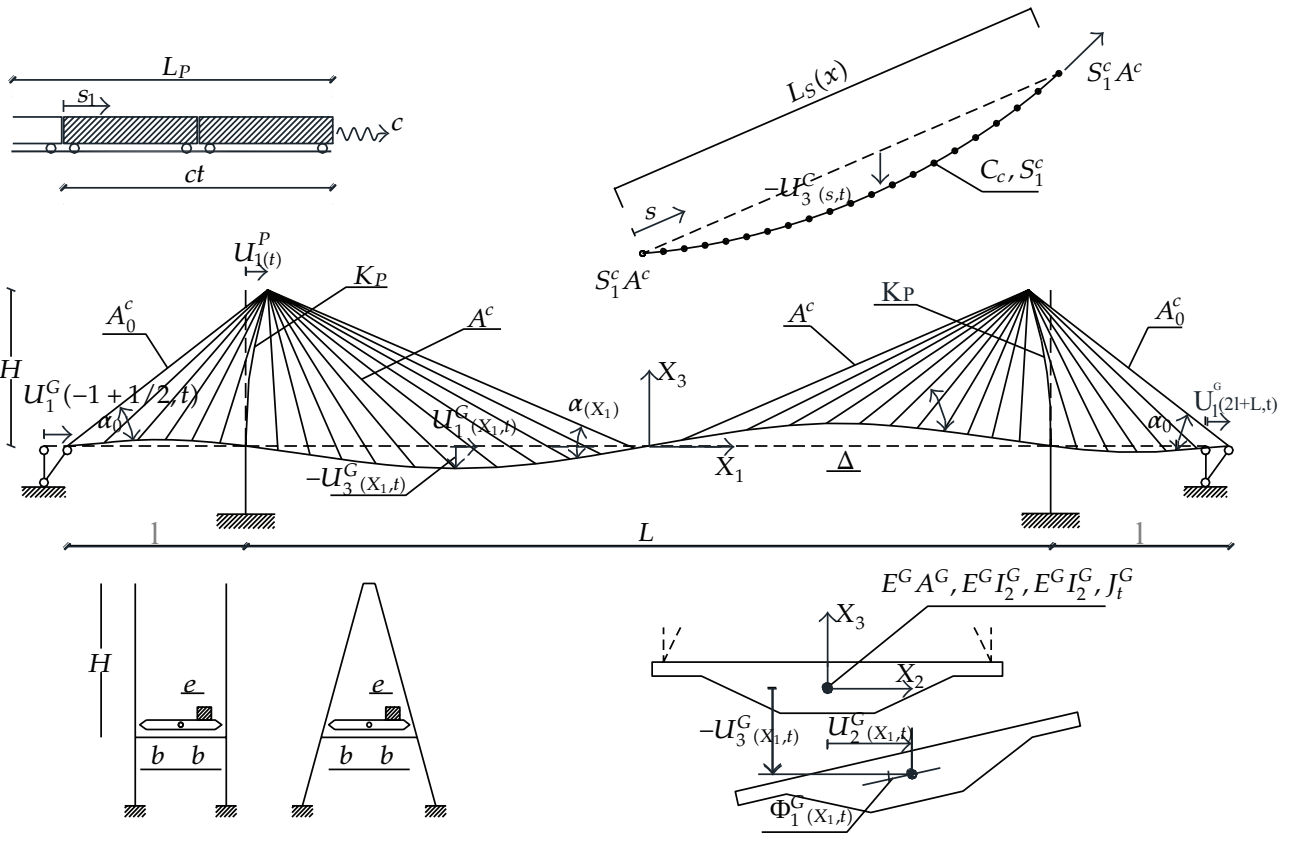

FIGURE 1: Cable-stayed bridge scheme: bridge kinematic, pylons, girder, and cable system characteristics.

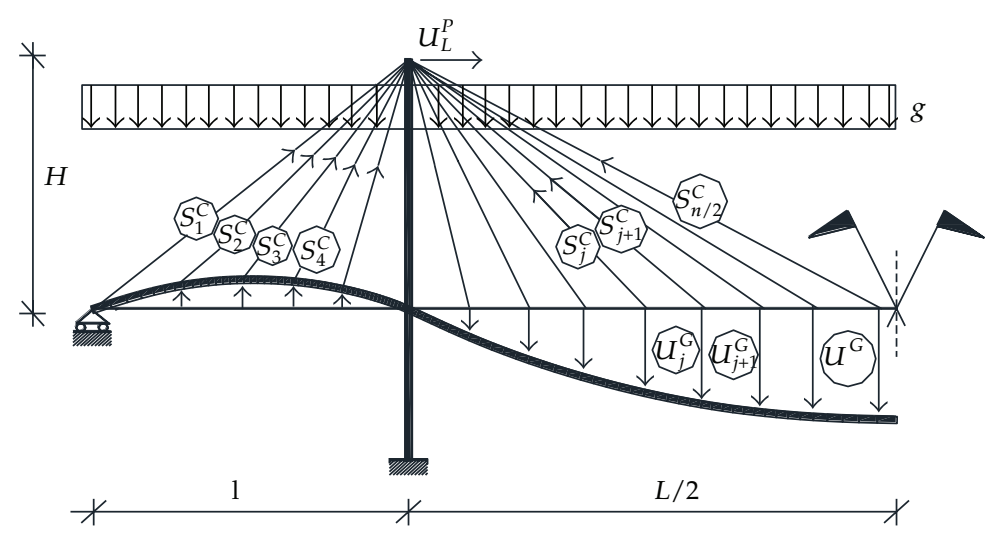

Figure 2: Cable-stayed configuration under dead loading: internal stresses and kinematic parameters.

elements, which are connected at the cross-section ends of the girder profile and at the top cross-section of the tower. The formulation is consistent with a large deformation theory based on the Green-Lagrange strain measure and the second Piola-Kirchhoff stress [20], whereas the material behavior is assumed to be linearly elastic. Since the cable behavior is mostly influenced by the preexisting stress and strain status, the initial configuration under the dead loading must be identified in advance. In particular, with respect to the final step of a balanced cantilever construction method, in which only the central segment of the deck is left, the geometric shape design of the bridge and the corresponding internal stresses of the cable system are obtained enforcing the deck and the top pylon cross-section kinematic variables to remain in the undeformed configuration, that is, "zero configuration" [10]. In order to calculate the initial stress distribution in the cable system, an optimization solving problem should be developed. In particular, with reference to the cable-stayed bridge scheme, reported in Figure 2, with $n$ number of stays, the objective functions are represented by the displacement vector $U_{\sim}$ containing the $n-2$ vertical displacements, that is, $\left(U_{2}^{G}, \ldots, U_{n-3}^{G}, U_{n-2}^{G}\right)$, excluding those points associated with the anchor stays at the cable/girder connections, and the horizontal displacements of the top cross-section of the pylons $\left(U_{L}^{P}, U_{R}^{P}\right)$ :

$$
U_{\sim}^{T}=\left[U_{L}^{P}, U_{R}^{P}, U_{2}^{G}, \ldots, U_{n-3}^{G}, U_{n-2}^{G}\right] .
$$

Moreover, the design variables, which have to be calculated, correspond to the internal stress distribution of the cable system, that is, ${\underset{\sim}{S}}^{T}=\left[S_{1}^{C}, S_{2}^{C}, \ldots, S_{n_{c}-1}^{C}, S_{n}^{C}\right]$. Since the relationships between displacements and cable stresses are essentially nonlinear, a specialized solving procedure to calculate the initial configuration is required. In particular, starting from an initial trial distribution in the cable system, that is, $S_{0}$, the vertical displacements under the dead loading 
can be expressed to the first order by the Taylor expansion in terms of the incremental cable stress distribution, by means of the following linearized equations:

$$
\underset{\sim}{U}\left(S_{\sim}+\Delta \underset{\sim}{S}, p\right)=\underset{\sim}{U}\left(S_{\sim}, p\right)+\left.\frac{d U_{\sim}}{d S}\right|_{\left(\mathcal{S}_{0}, p\right)} \cdot \Delta \underset{\sim}{S}+o\left\|\Delta S_{\sim}^{2}\right\|,
$$

where $\underset{\sim}{U}\left(S_{0}, p\right)$ is a vector containing the displacements in the self weight loading condition and subjected to the stress distribution $S_{0}, p$ is the loading parameter associated to the application of the dead loading, and $\left.\left(d \underset{\sim}{U} / d S_{\sim}\right)\right|_{(\underset{\sim}{0}, p)}$ is the directional derivative of $\underset{\sim}{U}$ at $S_{0}$ coinciding with the flexibility matrix of the structure. In (2), the unknown quantity is represented by the incremental vector related to the cable stress distribution, namely, $\Delta S$, which is determined

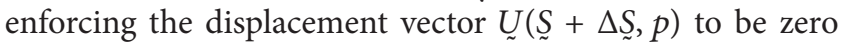
under the action of the dead loading. Since the structure is affected by a nonlinear behavior, an iterative procedure based on the Newton-Raphson scheme is adopted. More details regarding the solving procedure to calculate the zero configuration are reported in Section 3, which is, essentially, devoted to analyzing the numerical implementation of the proposed modeling.

With reference to the structural bridge scheme reported in Figure 1, it is assumed that the cable system is composed of undamaged elements and a fixed number of elements, which are affected during the moving loads by an internal cable failure mechanism. In particular, the damage formulation is developed by means of explicit time dependence laws, which reproduce the phenomenon of the cable release in a short time step due to an accidental failure in the cable system, such as the one occurring during a terroristic or vandalism attack and thus is not related to creep damage. On the contrary, the remaining stays are assumed to be undamaged and thus the damage function is set to zero. The stay failure of the $i$ th generic stay is simulated, by introducing a damage evolution law in the constitutive relationships. In particular, a time dependent damage function is utilized, which reduces, during the assumed failure time step, the elastic material properties of the cable as well as the corresponding initial internal stresses. Among the several approaches available from the literature, the formulation is assumed to be consistent with the Continuum Damage Mechanics Theory [21, 22]. In this context, degradation functions, physically based, are introduced to reproduce a typical damage phenomenon of the cable system. The definition of the damage function is developed introducing a scalar variable $D$ representative of the failure mechanism, defined by means of the following scalar expression:

$$
D(s, t)=\frac{k(s, t)-k_{d}(s, t)}{k(s, t)} \text { with } D \in[0,1],
$$

where $s$ is the curvilinear coordinate used to describe the arc length of the cable; $k$ and $k_{d}$ are the actual and residual stiffness properties, for example, area or modulus, of the cable, respectively. In order to reproduce the failure mechanism of the generic cable element, a time dependent damage law

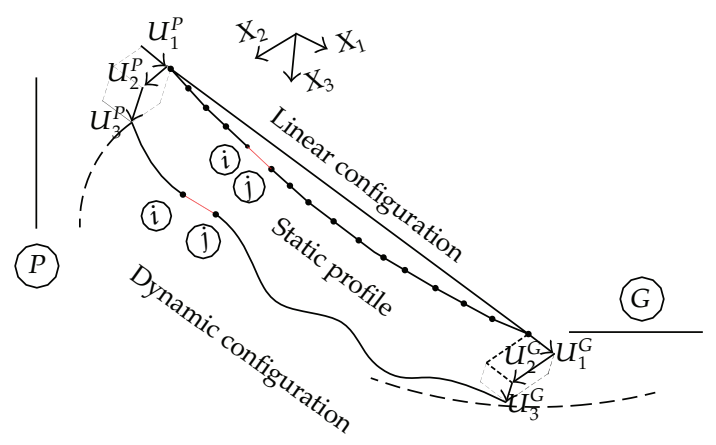

FIGURE 3: Initial and current configurations of the cable, support motion due to girder $(G)$, and pylon $(P)$ deformation.

based on Kachanov or Rabotnov type approach [21, 22] is introduced. The damage function is expressed through an explicit evolution law in the failure time domain, defined by the initial $\left(t_{0}\right)$ and the final $\left(t_{f}\right)$ times of the failure mechanism and the critical value of the damage variable $D_{c}$, by means of the following expression:

$$
D(s, t)=1-\left(1-\frac{t_{f}-\left(t-t_{0}\right)}{t_{f}}\right)^{1 /(1+m)},
$$

with

$$
t_{f}=\frac{1-\left(1-D_{c}\right)^{m+1}}{m+1}\left(\frac{S}{A_{0}}\right)^{-m}
$$

where $m$ and $A_{0}$ are material damage parameters. More details, about the mathematical relationships leading to (4)(5) can be found in the appendix. It is worth noting that the critical damage parameter represents the value corresponding to the occurrence of the complete failure of the cable element, in which the $i$ th generic stay does not have the possibility of transferring any internal stresses from the girder and the tower and thus can be considered not to produce any significant effects on the global behavior of the bridge. Moreover, the above formulation cannot be considered in the creep damage framework, since the cable release phenomenon is produced in a short time step and it is concerned to simulate, essentially, an accidental failure in the cable system.

The formulation is presented assuming that the cable element is deformed in its initial cable configuration under dead loading, that is, $\Omega_{c}$, and thus the deformed configuration of the cable due to the application of the live loads can be described by the following additive expression (Figure 3):

$$
\begin{aligned}
\underset{\sim}{\varphi}(\underset{\sim}{X}, t)= & \left(X_{1}+U_{1}^{C}(\underset{\sim}{X}, t)\right) n_{\sim}+\left(X_{2}+U_{2}^{C}(\underset{\sim}{X}, t)\right) n_{\sim} \\
& +\left(X_{3}+U_{3}^{C}(\underset{\sim}{X}, t)\right) n_{\sim},
\end{aligned}
$$

where $\underset{\sim}{X}$ with $\underset{\sim}{X}=\left[X_{1}, X_{2}, X_{3}\right]$ is the positional vector of the cable cross-sections $U_{i}^{C}$ with $i=1,3$ are the displacement components in the local reference axis $X_{i}$ described by the basis $n_{i}$ of the coordinate system. The main equations for 


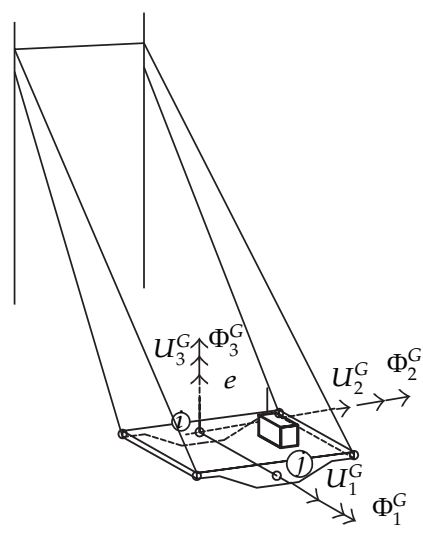

(a)

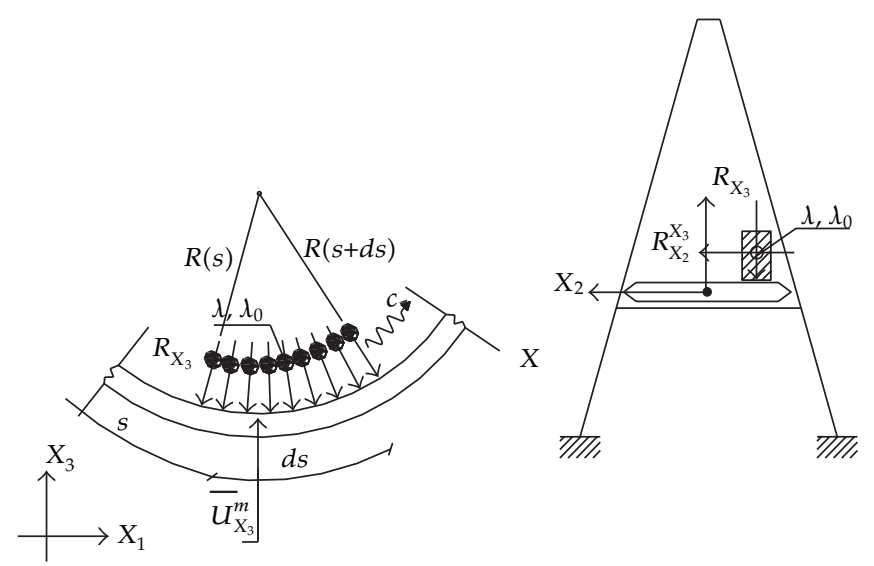

(b)

FIGURE 4: Girder kinematic (a) and moving loads description (b).

the generic $i$ th stay are described by introducing the damage evolution law defined by (4) in the constitutive law defined in terms of the Green-Lagrange strain and the second PiolaKirchhoff stress as

$$
\begin{gathered}
S_{1}^{C}(\underset{\sim}{X}, t)=C^{C}\left[1-\delta_{i k} D(t)\right] E_{1}^{C}(\underset{\sim}{X}, t)+S_{0}^{C}, \\
E_{1}^{C}(\underset{\sim}{X}, t)=U_{1, X_{1}}^{C}(\underset{\sim}{X}, t) \\
+\frac{1}{2}\left[U_{1, X_{1}}^{2}(\underset{\sim}{X}, t)+U_{2, X_{1}}^{2}(\underset{\sim}{X}, t)+U_{3, X_{1}}^{2}(\underset{\sim}{X}, t)\right]^{C},
\end{gathered}
$$

where $C^{C}$ is the elastic modulus, $\delta_{i k}$ is the Kronecker delta function, $S_{1}^{C}$ is the second Piola-Kirchhoff axial stress, $E_{1}^{C}$ is the Green-Lagrange axial strain, and $S_{0}^{C}$ is the second PiolaKirchhoff axial stress in the zero configuration. The governing equations of the motion of a single cable are expressed by means of the following partial differential equations [23]:

$$
\begin{aligned}
& \frac{d}{d X_{1}}\left[N_{1}^{C}+N_{1}^{C} \frac{d U_{1}^{C}}{d X_{1}}\right]-b_{1}-\mu_{c} \ddot{U}_{1}^{C}=0, \\
& \frac{d}{d X_{1}}\left[N_{1}^{C} \frac{d U_{2}^{C}}{d X_{1}}\right]-\mu_{c} \ddot{U}_{2}^{C}=0, \\
& \frac{d}{d X_{1}}\left[N_{1}^{C} \frac{d U_{3}^{C}}{d X_{1}}\right]-b_{2}-\mu_{c} \ddot{U}_{3}^{C}=0, \\
& \text { with } b_{1}=\mu_{G} g \cos \left(\varphi_{1}\right), \\
& \qquad b_{2}=\mu_{G} g \sin \left(\varphi_{2}\right),
\end{aligned}
$$

where $N_{1}^{C}$ is the axial force defined as $N_{1}^{C}=S_{1}^{C} A^{C}$ with $A^{C}$ the area of generic $i$ th cable element, $\varphi_{1}$ and $\varphi_{2}$ are the slope angles of the cable along the $X_{1} X_{2}$ and $X_{1} X_{3}$, respectively, and $b_{1}$ and $b_{2}$ are the body load projections in the $X_{1} X_{2}$ and $X_{1} X_{3}$, respectively. In addition to (8), boundary conditions on the cable kinematic are needed to describe the support motion produced by the girder and pylon motion at the corresponding intersection points with the cable development:

$$
\begin{array}{ll}
\bigcup_{\sim}^{C}\left(X_{0}\right)={\underset{\sim}{U}}^{P}, & \dot{\sim}^{C}\left({\underset{\sim}{0}}_{0}\right)=\dot{\sim}^{P}, \\
\bigcup_{\sim}^{C}\left(X_{\sim}\right)={\underset{\sim}{U}}^{G}, & \dot{\sim}^{C}\left({\underset{\sim}{0}}_{0}\right)=\dot{\sim}^{G},
\end{array}
$$

where $X_{0}$ and $X_{\sim}$ correspond to the initial and final crosssections of the cable element; $\left(U_{\sim}^{P}, \dot{U}^{P}\right)$ and $\left(U_{\sim}^{G}, \dot{U}^{G}\right)$ are the displacement and speed of the pylon $(P)$ and girder $(G)$, respectively, at the corresponding intersections with the cable development.

2.2. Girder and Moving Loads Interaction. Girder and towers are described by tridimensional geometric nonlinear beam elements by means of a formulation based on Euler-Bernoulli kinematic assumptions and a Green-Lagrange strain measure. The girder is connected with the cable system at the end points of the cable cross-sections and at the bottom crosssection ends of the towers, consistently defined with "H-" or "A-" shaped typologies. With reference to Figure 4(a), the displacements of the cross-section for a generic point located at the $\left(X_{1}, X_{2}, X_{3}\right)$ coordinate, that is, $\left(\bar{U}_{1}^{G}, \bar{U}_{2}^{G}, \bar{U}_{3}^{G}\right)$, are expressed by the following relationships:

$$
\begin{gathered}
\bar{U}_{1}^{G}\left(X_{1}, X_{2}, X_{3}, t\right)=U_{1}^{G}\left(X_{1}, t\right)+\Phi_{2}^{G}\left(X_{1}, t\right) X_{3} \\
-\Phi_{3}^{G}\left(X_{1}, t\right) X_{2}, \\
\bar{U}_{2}^{G}\left(X_{1}, t\right)=U_{2}^{G}\left(X_{1}, t\right), \\
\bar{U}_{3}^{G}\left(X_{1}, X_{2}, X_{3}, t\right)=U_{3}^{G}\left(X_{1}, t\right)+\Phi_{1}^{G}\left(X_{1}, t\right) X_{2},
\end{gathered}
$$

where $\left(U_{1}^{G}, U_{2}^{G}, U_{3}^{G}\right)$ and $\left(\Phi_{1}^{G}, \Phi_{2}^{G}, \Phi_{3}^{G}\right)$ are the displacement and rotation fields of the centroid axis of the girder with respect to the global reference system, respectively.

The external loads are assumed to proceed with constant speed $c$ from left to right along the bridge development and 
are supposed to be located, eccentrically with respect to the geometric axis of the girder. The moving system description refers to railway vehicle loads, which are reproduced by means equivalent uniformly distributed loads, perfectly connected to the girder profile. As a result, the kinematic parameters of the moving system coincide with the ones defined by the girder, neglecting frictional forces arising from the external loads, roughness effects of the girder profile, and local loading distribution produced by railway load components. However, these assumptions are quite recurrent in the framework of cable supported bridges with long spans, in which, typically, such interaction forces produced by localized dynamic effects are negligible with respect to the global bridge vibration [24]. Moreover, it is assumed that the damping energy is practically negligible. This hypothesis is verified in the context of long span bridges, where it has been proved that the bridge damping effects tend to decrease as span length increases $[25,26]$. Detailed results about the influence of damping effects on DAFs have been presented in $[5,6]$, from which it transpires that the assumption of an undamped bridge system leads to greater DAFs. With reference to the structural scheme reported in Figure 4(b), the infinitesimal reaction forces produced by the moving load on the girder profile can be expressed as a function of time dependent positional variable $s$, with $s(t)=c t$, by means of the balance of linear momentum, as follows:

$$
\begin{aligned}
& d R_{X_{3}}=\left.d X_{1}\left\{\lambda g+\frac{d}{d t}\left[\lambda \frac{d \dot{\bar{U}}_{3}^{m}}{d t}(s(t))\right]\right\}\right|_{s=X_{1}} \\
&=\left.d X_{1}\left\{\lambda g+\frac{d \lambda}{d t} \frac{d \dot{\bar{U}}_{3}^{m}}{d t}(s(t))+\lambda \frac{d^{2} \dot{\bar{U}}_{3}^{m}}{d t^{2}}(s(t))\right\}\right|_{s=X_{1}} \\
& d R_{X_{2}}=\left.d X_{1}\left\{\frac{d}{d t}\left[\lambda \frac{d \dot{\bar{U}}_{2}^{m}}{d t}(s(t))\right]\right\}\right|_{s=X_{1}} \\
&=\left.d X_{1}\left\{\frac{d \lambda}{d t} \frac{d \dot{\bar{U}}_{2}^{m}}{d t}(s(t))+\lambda \frac{d^{2} \dot{\bar{U}}_{2}^{m}}{d t^{2}}(s(t))\right\}\right|_{s=X_{1}}, \\
&\left.d R_{X_{1}}=\left.d X_{1}\left\{\frac{d}{d t}\left[\lambda \frac{d \dot{\bar{U}}_{1}^{m}}{d t}(s(t))\right]\right\}\right|_{s=X_{1}} \dot{\bar{U}}_{1}^{m}(s(t))\right\}\left.\right|_{s=X_{1}}, \\
&=d X_{1}\left\{\frac{d \lambda}{d t} \frac{\dot{\bar{U}}_{1}^{m}}{d t}(s(t))+\lambda \frac{d^{2}}{d t^{2}}\right.
\end{aligned}
$$

where $g$ is the gravitational acceleration, $\lambda$ is the external mass per unit length, and $\bar{U}_{i}^{m}$ with $i=1,3$ are the displacement functions along $X_{i}$ axis of the moving mass, identified by the girder kinematic by using (10), as $\bar{U}_{i}^{m}=\bar{U}_{i}$. It is worth noting that in (11).1, at right hand side, the first term represents the dead loading contribution, whereas, the second term is produced by the unsteady mass distribution in the system due to time dependence character of the mass function arising from the moving loads. Finally, the third term must be calculated taking into account the relative motion between bridge and the moving mass as follows [27]:

$$
\begin{gathered}
\frac{d \bar{U}_{i}^{m}}{d t}=\frac{\partial \bar{U}_{i}^{m}}{\partial t}+\frac{\partial \bar{U}_{i}^{m}}{\partial s} \frac{\partial s}{\partial t} \\
=\frac{\partial \bar{U}_{i}^{m}}{\partial t}+\frac{\partial \bar{U}_{i}^{m}}{\partial s} c \\
\frac{d^{2} \bar{U}_{i}^{m}}{d t^{2}}=\frac{d}{d t}\left[\frac{\partial \bar{U}_{i}^{m}}{\partial t}+\frac{\partial \bar{U}_{i}^{m}}{\partial t} \frac{\partial s(t)}{\partial t}\right] \\
=\frac{\partial^{2} \bar{U}_{i}^{m}}{\partial t^{2}}+2 c \frac{\partial^{2} \bar{U}_{i}^{m}}{\partial t \partial s}+c^{2} \frac{\partial^{2} \bar{U}_{i}^{m}}{\partial s^{2}}
\end{gathered}
$$

It is worth noting that the Eulerian description of the moving system introduces in (13) three terms corresponding to standard, centripetal, and Coriolis acceleration functions, respectively. However, the last two contributions in the acceleration function for the transverse and longitudinal displacements, that is, when $i=1,2$, are typically negligible in comparison to the term associated with the standard acceleration and thus they are not considered in the following computations. Substituting (10) into (11) with $\bar{U}_{i}^{m}=\bar{U}_{i}$, and making the use of (12)-(13), the reaction forces per unit length produced by the moving system are described by the following expressions:

$$
\begin{aligned}
p_{X_{3}}= & \frac{d R_{X_{3}}}{d X_{1}} \\
= & \lambda g+\frac{d \lambda}{d t}\left[\left(\frac{\partial U_{3}^{G}}{\partial t}+e \frac{\partial \Phi_{1}^{G}}{\partial t}\right)+c\left(\frac{\partial U_{3}^{G}}{\partial X_{1}}+e \frac{\partial \Phi_{1}^{G}}{\partial X_{1}}\right)\right] \\
& +\lambda\left[\frac{\partial^{2} U_{3}^{G}}{\partial t^{2}}+2 c \frac{\partial^{2} U_{3}^{G}}{\partial t \partial X_{1}}+c \frac{\partial^{2} U_{3}^{G}}{\partial X_{1}^{2}}\right] \\
& +\lambda \cdot e\left[\frac{\partial^{2} \Phi_{1}^{G}}{\partial t^{2}}+2 c \frac{\partial^{2} \Phi_{1}^{G}}{\partial t \partial X_{1}}+c \frac{\partial^{2} \Phi_{1}^{G}}{\partial X_{1}^{2}}\right] \\
p_{X_{2}}= & \frac{d R_{X_{2}}}{d X_{1}}=\frac{d \lambda}{d t} \frac{\partial U_{2}^{G}}{\partial t}+\lambda \frac{\partial^{2} U_{2}^{G}}{\partial t^{2}}, \\
p_{X_{1}}= & \frac{d R_{X_{1}}}{d X_{1}} \\
= & \frac{d \lambda}{d t}\left[\left(\frac{\partial U_{1}^{G}}{\partial t}-e \frac{\partial \Phi_{3}^{G}}{\partial t}\right)\right]+\lambda \frac{\partial^{2} U_{1}^{G}}{\partial t^{2}}-\lambda \cdot e \frac{\partial^{2} \Phi_{3}^{G}}{\partial t^{2}} \\
= & \frac{d \lambda}{d t}\left[\left(\frac{\partial U_{1}^{G}}{\partial t}-e \frac{\partial^{2} U_{2}^{G}}{\partial t \partial X_{1}}\right)\right]+\lambda \frac{\partial^{2} U_{1}^{G}}{\partial t^{2}}-\lambda \cdot e \frac{\partial^{3} U_{3}^{G}}{\partial t^{2} \partial X_{1}}
\end{aligned}
$$

where $e$ is the eccentricity of the moving loads with respect to the girder geometric axis. Moreover, in (14), the mass function during the external load advance can be expressed 
with respect to the global reference system assumed from the left end of the bridge as

$$
\lambda\left(s_{1}, t\right)=\lambda_{\mathrm{ML}} \bar{H}\left(s_{1}+L_{p}-c t\right) \bar{H}\left(c t-s_{1}\right),
$$

where $\bar{H}(\cdot)$ is the Heaviside step function, $L_{p}$ is the length of the moving loads, $s_{1}$ is the referential coordinate located at the left end of the girder cross-section, that is, $s_{1}=l+$ $L / 2+X_{1}$, and $\lambda_{M}$ is the mass linear density of the moving system.

The kinematic model is consistent with the geometric nonlinear Euler-Bernoulli theory, in which moderately large rotations are considered [26]. The torsional behavior owing to eccentric loading is described by means of the classical De Saint Venant theory. In particular, the strains are based on the Green-Lagrange measure in which only the square of the terms $U_{i, X_{1}}^{2 G}$ representing the rotations of the transverse normal line in the beam is considered. Therefore, starting from (10), the following relationships between generalized strain and stress variables are obtained:

$$
\begin{aligned}
N_{1}^{G} & =N_{1}^{0(G)}+E A^{G} \varepsilon_{1}^{G} \\
& =N_{1}^{0(G)}+E^{G} A^{G}\left\{U_{1, X_{1}}^{G}+\frac{1}{2}\left[U_{1, X_{1}}^{2 G}+U_{2, X_{1}}^{2 G}+U_{3, X_{1}}^{2 G}\right]\right\} \\
M_{2}^{G} & =M_{2}^{0(G)}+E I_{2}^{G} \chi_{2}^{G} \\
& =M_{2}^{0(G)}+E^{G} I_{2}^{G} \Phi_{2, X_{1}}^{G} \\
& =M_{2}^{0(G)}-E I_{2}^{G} U_{3, X_{1} X_{1}}^{G}, \\
M_{3}^{G} & =M_{3}^{0(G)}+E I_{3}^{G} \chi_{3}^{G} \\
& =M_{3}^{0(G)}+E^{G} I_{3}^{G} \Phi_{3, X_{1}}^{G} \\
& =M_{3}^{0(G)}+E I_{3}^{G} U_{2, X_{1} X_{1}}^{G}, \\
M_{1}^{G} & =G J_{t}^{G} \Theta^{G}=G^{G} J_{t}^{G} \Phi_{1, X_{1}}^{G},
\end{aligned}
$$

where $E A^{G}$ and $\varepsilon_{1}^{G}$ are the axial stiffness and strain, $\chi_{2}^{G}$ and $\chi_{3}^{G}$ or $E^{G} I_{2}^{G}$ and $E^{G} I_{3}^{G}$ are the curvatures or the bending stiffnesses with respect to the $X_{2}$ and $X_{3}$ axes, respectively, $\Theta^{G}$ and $G^{G} J_{t}^{G}$ are the torsional curvature and stiffness, respectively, $N_{1}^{G}$ is the axial stress resultant, $M_{2}^{G}$ and $M_{3}^{G}$ are the bending moments with respect to the $X_{2}$ and $X_{3}$ axes, respectively, $M_{1}^{G}$ and $G^{G} J_{t}^{G}$ are torsional moment and girder stiffness, respectively, and $(\cdot)^{0}$ represents the superscript concerning the variables associated with the "zero configuration." On the basis of (16), taking into account of (14)-(15) and notation reported in Figure 4(a), the following governing equations are derived by means of the local form of dynamic equilibrium equations:

$$
\begin{gathered}
\frac{d}{d X_{1}}\left\{N_{1}^{G}\left(1+\frac{d U_{1}^{G}}{d X_{1}}\right)\right\}-\mu_{G} \ddot{U}_{1}^{G}+p_{X_{1}}=0, \\
-E^{G} I_{2}^{G} \frac{d^{4} U_{3}^{G}}{d X_{1}^{4}}-\frac{d}{d X_{1}}\left(N_{1}^{G} \frac{d U_{3}^{G}}{d X_{1}}\right) \\
-\mu_{G} \ddot{U}_{3}^{G}-\ddot{\Phi}_{2, X 1}^{G} I_{02}^{G}-p_{X_{3}}=0, \\
E^{G} I_{3}^{G} \frac{d^{4} U_{2}^{G}}{d X_{1}^{4}}-\frac{d}{d X_{1}}\left(N_{1}^{G} \frac{d U_{2}^{G}}{d X_{1}}\right) \\
-\mu_{G} \ddot{U}_{2}^{G}-\ddot{\Phi}_{3, X 1}^{G} I_{03}^{G}+p_{X_{2}}=0, \\
G^{G} J_{t}^{G} \frac{d^{2} \Phi_{1}^{G}}{d X_{1}^{2}}-\left(I_{01}^{G}+\lambda_{\mathrm{ML}}^{0}\right) \ddot{\Phi}_{1}^{G}-p_{X_{3}} e=0,
\end{gathered}
$$

where $\lambda_{\mathrm{ML}}^{0}$ is the per unit length torsional girder mass; $\mu_{G}$ is the girder mass per unit length. Additional equations are required to take into account interelement continuity and initial conditions concerned with solving the dynamic problem, which can be expressed with reference to the $i$ th girder element as follows:

$$
\begin{array}{ll}
U_{k}^{G}(0)=0, & \dot{U}_{k}^{G}(0)=0, \\
U_{k}^{i(G)}(0)=U_{k}^{i-1(G)}\left(l_{e}^{i-1}\right), \quad & U_{k}^{G}\left(l_{e}^{i}\right)=U_{k}^{i+1}(0), \\
\Phi_{k}^{G}(0)=0, \quad \dot{\Phi}_{k}^{G}(0)=0, & \\
\Phi_{k}^{i(G)}(0)=\Phi_{k}^{i-1(G)}\left(l_{e}^{i-1}\right), \quad & \Phi_{k}^{G}\left(l_{e}^{i}\right)=\Phi_{k}^{i+1}(0),
\end{array}
$$

where the superscripts $i+1$ and $i-1$ indicate the previous or the next girder elements and the subscript $k$, with $k=1,2,3$ defines the displacement and rotation directions with respect to the coordinate reference system.

\section{Finite Element Implementation}

The governing equations reported in the previous section introduce a nonlinear partial differential system, whose analytical solution is quite complex to be extracted. As a consequence, a numerical approach based on the finite element formulation is utilized. In particular, starting from (8) and (17), the corresponding weak forms for the ith finite element related to the girder $(G)$ and the cable system $(C)$, respectively, are defined by the following expressions.

\section{Girder}

$$
\begin{aligned}
& \int_{l_{e}^{i}} N_{1}^{G}\left(1+U_{1, X_{1}}^{G}\right) w_{1, X_{1}} d X_{1}-\mu_{g} \int_{l_{e}^{i}} \ddot{U}_{1}^{G} w_{1} d X_{1} \\
& -\lambda_{\mathrm{ML}} \int_{l_{e}^{i}}\left[-\bar{\delta}_{1}+\bar{\delta}_{2}\right] \dot{U}_{1}^{G} w_{2} d X_{1}-\lambda_{\mathrm{ML}} \int_{l_{e}^{i}} \bar{H}_{1} \bar{H}_{2} \ddot{U}_{1}^{G} w_{2} d X_{1} \\
& \quad-\sum_{j=1}^{2} N_{1 j}^{G} U_{1 j}^{G}=0
\end{aligned}
$$




$$
\begin{aligned}
& \int_{l_{e}^{i}}\left\{-M_{2}^{G} w_{2, X_{1} X_{1}}+\left(N_{1}^{G} U_{3, X_{1}}^{G}\right) w_{2, X_{1}}\right\} d X_{1} \\
& -\mu_{g} \int_{l_{e}^{i}} \ddot{U}_{3}^{G} w_{2} d X_{1}+I_{02}^{G} \int_{l_{e}^{i}} \ddot{U}_{3, X_{1}}^{G} w_{2} d X_{1} \\
& -\lambda_{\mathrm{ML}} \int_{l_{e}^{i}}\left[-\bar{\delta}_{1}+\bar{\delta}_{2}\right]\left(\dot{U}_{3}^{G}+c U_{3, X_{1}}^{G}\right) w_{2} d X_{1} \\
& -\lambda_{\mathrm{ML}} \int_{l_{e}^{i}} \bar{H}_{1} \bar{H}_{2}\left[\left(\ddot{U}_{3}^{G}+2 c \dot{U}_{3, X_{1}}^{G}+c^{2} U_{3, X_{1} X_{1}}^{G}\right)+g\right] w_{2} d X_{1} \\
& -\sum_{j=1}^{2} T_{3 j}^{G} U_{3 j}^{G}-\sum_{j=1}^{2} M_{2 j}^{G} \Phi_{3 j}^{G}=0, \\
& \int_{l_{e}^{i}}\left\{M_{3}^{G} w_{3, X_{1} X_{1}}+\left(N_{1}^{G} U_{2, X_{1}}^{G}\right) w_{3, X_{1}}\right\} d X_{1} \\
& -\mu_{g} \int_{l_{e}^{i}} \ddot{U}_{2}^{G} w_{3} d X_{1}-I_{03}^{G} \int_{l_{e}^{i}} \ddot{U}_{2, X_{1}}^{G} w_{3} d X_{1} \\
& -\lambda_{\mathrm{ML}} \int_{l_{e}^{i}}\left[-\bar{\delta}_{1}+\bar{\delta}_{2}\right] \dot{U}_{2}^{G} w_{2} d X_{1} \\
& -\int_{l_{e}^{i}} \bar{H}_{1} \bar{H}_{2}\left[\ddot{U}_{2}^{G}+g-e \ddot{U}_{2, X 1}^{G}\right] w_{2} d X_{1} \\
& -\lambda_{\mathrm{ML}} e \int_{l_{e}^{i}}\left[-\bar{\delta}_{1}+\bar{\delta}_{2}\right] \dot{U}_{2, X_{1}}^{G} w_{2} d X_{1} \\
& -\sum_{j=1}^{2} T_{2 j}^{G} U_{2 j}^{G}-\sum_{j=1}^{2} M_{3 j}^{G} \Phi_{2 j}^{G}=0, \\
& \int_{l_{e}^{i}} M_{1}^{G} w_{4, X_{1}} d X_{1}-I_{01} \int_{l_{e}^{i}} \ddot{\Phi}_{1}^{G} w_{4} d X_{1} \\
& +\lambda_{\mathrm{ML}}\left(e+\frac{\lambda_{\mathrm{ML}}^{0}}{\lambda_{\mathrm{ML}}}\right) \\
& \times \int_{l_{e}^{i}} \bar{H}_{1} \bar{H}_{2}\left(\ddot{\Phi}_{1}^{G}+2 c \dot{\Phi}_{1, X_{1}}^{G}+c^{2} \Phi_{1, X_{1} X_{1}}^{G}\right) w_{4} d X_{1} \\
& -\lambda_{M L}\left(e+\frac{\lambda_{\mathrm{ML}}^{0}}{\lambda_{\mathrm{ML}}}\right) \int_{l_{e}^{i}}\left[-\bar{\delta}_{1}+\bar{\delta}_{2}\right]\left(\dot{\Phi}_{1}^{G}+c \Phi_{1, X_{1}}^{G}\right) w_{4} d X_{1} \\
& -\sum_{j=1}^{2} M_{1 j}^{G} \Phi_{1 j}^{G}=0 \text {. }
\end{aligned}
$$

Cable System

$$
\begin{aligned}
& \int_{l_{e}^{i}} N_{1}^{C}\left(1+U_{1, X 1}^{C}\right) w_{1, X_{1}} d X_{1}-\mu_{c} \int_{l_{e}^{i}} \ddot{U}_{1}^{C} w_{1} d X_{1} \\
& -\int_{l_{e}^{i}} b_{1} w_{1} d X_{1}-\sum_{j=1}^{2} N_{1 j} U_{1 j}^{C}=0, \\
& \int_{l_{e}^{i}} N_{1}^{C} w_{2, X_{1}} d X_{1}-\mu_{c} \int_{l_{e}^{i}} \ddot{U}_{2}^{C} w_{2} d X_{1}-\sum_{j=1}^{2} T_{2 j}^{C} U_{2 j}^{C}=0, \\
& \int_{l_{e}^{i}} N_{1}^{C} w_{3, X_{1}} d X_{1}-\mu_{c} \int_{l_{e}^{i}} \ddot{U}_{3}^{C} w_{3} d X_{1} \\
& \quad-\int_{l_{e}^{i}} b_{3} w_{3} d X_{1}-\sum_{j=1}^{2} T_{3 j}^{C} U_{3 j}^{C}=0,
\end{aligned}
$$

where $\bar{H}_{1}=H\left(s_{1}+L_{p}-c t\right), \bar{H}_{2}=H\left(c t-s_{1}\right), \bar{\delta}_{1}=$ $\delta\left(s_{1}+L_{p}-c t\right), \bar{\delta}_{2}=\delta\left(c t-s_{1}\right), \delta(\cdot)$ represents the delta Dirac functions, $\left(N_{1 i}, T_{2 i}, T_{3 i}, M_{2 i}, M_{3 i}\right)^{k}$ with $k=C, G$ and $i=1,2$ represents the internal forces applied at the end node $i$ of the generic cable $(C)$ or girder $(G)$ element. Moreover, the pylon governing equations can be easily obtained from (19), by removing all the terms related to the moving loads and changing the relative variables from the superscript $(\cdot)^{G}$ to $(\cdot)^{P}$ and the parameters concerning the mechanical and material characteristics. As a consequence, for conciseness, the governing equations concerning the pylon dynamic behavior are not reported.

Finite element expressions are written starting from the weak forms previously reported, introducing Hermit cubic interpolation functions $\left(\xi_{i}\right)$ for the girder and pylon flexures in the $X_{1} X_{2}$ and $X_{2} X_{3}$ deformation planes and Lagrange linear interpolation functions $\left(\zeta_{i}\right)$ for the cable system variables and the remaining variables of the girder and the pylons:

$$
\begin{gathered}
{\underset{\sim}{C}}^{C}(\underset{\sim}{r}, t)=N_{\sim}^{C}(\underset{\sim}{r}){\underset{\sim}{q}}^{C}(t), \quad{\underset{\sim}{U}}^{G}(\underset{\sim}{r}, t)=N_{\sim}^{G} q_{\sim}^{G}(t), \\
{\underset{\sim}{U}}^{P}(\underset{\sim}{r}, t)=N_{\sim}^{N^{P}} \underline{\sim}^{P}(t),
\end{gathered}
$$

where $q_{C}$ and $q_{G}$ are the vectors collecting the nodal degrees of freedom of the cable, girder respectively, $N_{C}, N_{N}$, and $N_{P}$ are the matrixes containing the displacement interpolation function for cable element $(C)$, girder $(G)$, and pylons $(P)$, and $r$ is the local coordinate vector of the $i$ th finite element. The discrete equations in the local reference system of the $i$ th element are derived substituting (21) into (19)-(20), leading to the following equations in matrix notation:

$$
\begin{aligned}
& \left(M_{\sim}^{G}+M_{\sim \mathrm{NS}}^{G}\right) \ddot{\sim}^{G}+\left(C_{\sim}^{G}+{\underset{\sim}{\mathrm{NS}}}_{C_{\sim}}\right) \dot{\sim}^{G}
\end{aligned}
$$

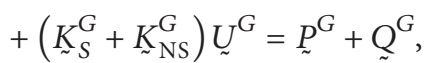

$$
\begin{aligned}
& M_{\sim}^{P} \ddot{U}^{P}+C_{\sim}^{P} \dot{U}^{P}+K_{\sim}^{P}{\underset{\sim}{U}}^{P}=P_{\sim}^{P}+Q_{\sim}^{P}, \\
& M_{\sim}^{C} \ddot{\sim}^{C}+C_{\sim}^{C} \dot{\sim}^{C}+K_{\sim}^{C}{\underset{\sim}{C}}^{C}={\underset{\sim}{C}}^{C}+Q^{C},
\end{aligned}
$$

where $M_{\sim}$ is the mass matrix, $C_{i}$ is the damping matrix, $K_{i}$ is the stiffness matrix, $P_{i}$ is the load vector produced by the dead and live loading, $Q_{i}$ is the unknown force vector collecting the point sources, and the subscripts $(\cdot)_{S}$ or $(\cdot)_{\mathrm{NS}}$ refer to standard or nonstandard terms, respectively, introduced in the discrete equations. Most of the matrixes reported in (22) can be easily recovered from the literature [26]. Contrarily, the matrixes ${\underset{\sim}{N S}}_{N S}, C_{N S}$, and $K_{N S}$ collect the nonstandard terms arising from the inertial description of the live loads and the interaction behavior between moving loads and bridge motion and are defined by the following expressions:

$$
\begin{aligned}
& \left(M_{\sim}^{G}\right)=\int_{l_{e}^{i}} \bar{H}_{1} \bar{H}_{2}\left(N_{\sim}^{G(T)} \Lambda_{\sim} M_{1} N_{\sim}^{G}\right) d X_{1}, \\
& \left(C_{\mathrm{NS}}^{G}\right)_{i j}=\int_{l_{e}^{i}}\left[\left(-\bar{\delta}_{1}+\bar{\delta}_{2}\right)\left(N_{\sim}^{G(T)}{ }_{\sim}{ }_{C_{1}} N_{\sim}^{G}\right)\right. \\
& \left.+\bar{H}_{1} \bar{H}_{2}\left(N_{\sim}^{G(T)} X_{1} \Lambda_{\sim} C_{2} N_{\sim}^{G}\right)\right] d X_{1},
\end{aligned}
$$




$$
\begin{aligned}
\left(K_{\sim \mathrm{NS}}^{G}\right)_{i j}=\int_{i_{e}^{i}}[ & \left(-\bar{\delta}_{1}+\bar{\delta}_{2}\right)\left(\underset{\sim 1, X_{1}}{N_{1}^{G(T)}} \Lambda_{K_{1}} N_{\sim}^{G}\right) \\
& \left.+\bar{H}_{1} \bar{H}_{2}\left(N_{\sim}, X_{1} X_{1}(T) \Lambda_{K_{2}} N_{\sim}^{G}\right)\right] d X_{1},
\end{aligned}
$$

where the matrixes $\Lambda_{M_{1}}, \Lambda_{C_{1,2}}$, and $\Lambda_{K_{1,2}}$, which assemble the coefficients associated with the inertial contribution arising from the moving loads and girder interaction, are defined as:

$$
\begin{aligned}
& \Lambda_{M_{1}}=\operatorname{diag}\left[\lambda_{\mathrm{ML}}, \lambda_{\mathrm{ML}}, \lambda_{\mathrm{ML}}, \lambda_{\mathrm{ML}} e+\lambda_{\mathrm{ML}}^{0}, 0,0\right], \\
& \Lambda_{M_{1}}=\operatorname{diag}\left[0,-e \lambda_{\mathrm{ML}}, 0,0,0,0\right], \\
& \Lambda_{C_{1}}=\operatorname{diag}\left[\lambda_{\mathrm{ML}}, \lambda_{\mathrm{ML}}, \lambda_{\mathrm{ML}}, \lambda_{\mathrm{ML}} e+\lambda_{\mathrm{ML}}^{0}, 0,-\lambda_{\mathrm{ML}} e\right], \\
& \Lambda_{\mathrm{C}_{2}}=\operatorname{diag}\left[0,0,2 c \lambda_{\mathrm{ML}}, 2 c\left(\lambda_{\mathrm{ML}} e+\lambda_{\mathrm{ML}}^{0}\right), 0,0\right], \\
& \Lambda_{K_{1}}=\operatorname{diag}\left[0,0, c \lambda_{\mathrm{ML}}, c\left(\lambda_{\mathrm{ML}} e+\lambda_{\mathrm{ML}}^{0}\right), 0,0\right], \\
& \Lambda_{K_{2}}=\operatorname{diag}\left[0,0, c^{2} \lambda_{\mathrm{ML}}, c^{2}\left(\lambda_{\mathrm{ML}} e+\lambda_{\mathrm{ML}}^{0}\right), 0,0\right] .
\end{aligned}
$$

In order to reproduce the bridge kinematic correctly, additional relationships to define the connections between girder, pylon, and cable system are necessary. In particular, the cable system displacements should be equal to those of the girder and the pylons at the corresponding intersection points; thus, the bridge kinematic is restricted by means of the following constrain equations:

$$
\begin{aligned}
& U_{3}^{G}\left(\underset{\sim}{X_{C_{i}}}, t\right)+\Phi_{1}^{G}\left(\underset{\sim}{X} C_{C_{i}}, t\right) b=U_{3}^{C}\left({\underset{\sim}{C} i}_{C_{i}}, t\right), \\
& U_{1}^{G}\left(\underset{\sim}{X_{C_{i}}}, t\right)-\Phi_{3}^{G}\left({\underset{\sim}{X} C_{i}}_{C^{\prime}}, t\right) b=U_{1}^{C}\left(\sim_{C_{i}}, t\right) \text {, } \\
& U_{1}^{P}(\underset{\sim}{X}, t)=U_{1}^{\mathrm{C}}\left(\underset{\sim}{X_{P}}, t\right), \\
& U_{2}^{P}(\underset{\sim}{X}, t)=U_{2}^{C}(\underset{\sim}{X}, t), \\
& U_{3}^{P}(\underset{\sim}{X}, t)=U_{3}^{\mathrm{C}}(\underset{\sim}{X}, t),
\end{aligned}
$$

where ${\underset{\sim}{X}}_{C_{i}}$ and $\underset{\sim}{X}$ represent the vectors containing the intersection positions of the $i$ th cable element and the pylon top cross-section, respectively. Finally, starting from (22), taking into account (25) as well as the balance of secondary variables at the interelement boundaries, the resulting equations of the finite element model are

$$
M \underset{\sim}{Q}+\underset{\sim}{C} \dot{Q}+K_{\sim} Q_{\sim}=\underset{\sim}{P},
$$

where $Q$ with $Q={\underset{\sim}{U}}_{B} \cup{\underset{\sim}{G}}_{G} \cup U_{\sim}$ is the generalized coordinate vector containing the kinematic variables associated with the girder, the pylons, and the cable system, $M, C$, and $K$ are the global mass, stiffness, and damping matrixes, and $\underset{\tilde{n}}{P}$ is the loading vector. Since the structural behavior of each element depends on the deformation state of the members, the governing equations defined by (26) will change continuously as the structure deforms. Moreover, the external loads owing to the presence of its own moving mass determine a time dependent mass distribution function on the girder profile. Consequently, the discrete equations are affected by nonlinearities in the stiffness matrix and time dependence in the mass matrix.

The governing equations are solved numerically, using a user customized finite element program, that is, COMSOL Multiphysics TM version 4.1 [27]. The analysis is performed by means of two different stages. Initially, a preliminary analysis is devoted to calculating the initial stress distribution in the cable system, that is, "zero configuration." In this context, the shape optimization procedure is developed, consistently with a Newton-Raphson iteration scheme. Since the loading condition refers to the application of dead loading only, the analysis is developed in the framework of a static analysis. In particular, with reference to (2), at the $k$ th iteration, the incremental value of the cable system stresses $\Delta S_{k}$ is determined by neglecting the second order residuum obtained by the Taylor expansion, that is, $o\left\|\Delta S_{k}^{2}\right\|$ and by solving the linear equation system enforcing the bridge deformations to verify the constraint equations given by the zero configuration, namely, $U_{\sim}\left(S_{k}+\Delta S_{k}, \lambda\right)=0$, as.

$$
\Delta S_{k}=-\left[\left.\frac{d U_{\tilde{N}}}{d S_{\sim}}\right|_{\left(S_{k}, \lambda\right)} ^{-1}\right]_{k}^{U}\left(S_{k}, \lambda\right) .
$$

The iterative values of the cable stresses are determined from (27) as $S_{k+1}=S_{k}+\Delta S_{k}$, whereas the final quantities are obtained by means of an incremental-iterative procedure, which is developed on the basis of a tolerance convergence criterion. In particular, the residual norm is checked to be lower than a fixed prescribed tolerance, that is, $\| U_{\sim}\left(S_{k}+\right.$ $\left.\Delta S_{k}, \lambda\right) \| \leq$ toll; if it is not verified, new values of the stresses are determined by means of an iterative scheme defined on the basis of (27). Once the initial configuration is determined in terms of the initial cable stress and strain distribution, a sequential dynamic analysis is developed. Since the governing equations introduce essentially a time dependent nonlinear equation system, an incremental and iterative integration scheme is required. The algebraic equations are solved by a direct integration method, which is based on an implicit time integration scheme. In particular, an implicit temporal discretization of order two using a Backward Differentiation Formula (BDF-2) with an adaptative time step is utilized. Moreover, a Newton-Raphson scheme in the time step increment based on the secant formulation is utilized for the nonlinearities involved in the governing equations [28]. In order to guarantee accuracy in the predicted results, particular attention is devoted to the choice of the time integration step, which, assuming small vibrations about the nonlinear equilibrium configuration under dead loads, can be defined as a function of the periods of those vibration mode shapes having a relevant participation on the response. However, in the case of moving load excitation, the dynamic solution strongly depends on the speed of the moving system, since different vibration frequencies are activated for low or large transit speeds [27]. In the present analyses, the initial integration time step, which is automatically reduced due to the time adaptation procedure, is assumed as at least 1/1000 of the observation period defined as the time necessary for the moving train to cross the bridge. This value turns out to 
be always lower than $1 / 100$ of the 50 th natural period of the bridge structure, the first natural period being the largest one.

\section{Results}

Results are proposed to investigate the behavior of cablestayed bridge subjected to an accidental failure in the cable system. From the design point of view, existing codes on this argument, that is, PTI [15] and SETRA [16], recommend to reproduce the effects of such loading scheme by performing a quasistatic analysis and taking into account the dynamic amplification effects by means of amplification factors in the range between 1.5-2.0. For this reason, the purpose of the following results is to identify the dynamic amplification effects produced by the cable failure by using a refined dynamical formulation in place of a simplified quasi-static one. In order to quantify the amplification effects produced by the moving loads over the static solution, numerical results are proposed in terms of dynamic amplification factors for undamaged (UD) and damaged $(D)$ cable-stayed structures in terms of the moving loads and the bridge characteristics. It is worth noting that UD cable-stayed configurations refer to a bridge structures, in which the stays are not affected by any damage mechanisms. Contrarily, damaged $(D)$ cable-stayed scheme corresponds to bridge configurations, in which one or more stays are subjected to the explicit damage mechanism defined on the basis of (3). Moreover, in the following results, the cross-section exposed to damage is assumed to be located at the intersection between the stay and girder. The dynamic amplification factors (DAFs) for the generic variable $X$ under investigation related to damaged or undamaged structural configuration, namely, $\Phi_{X}^{D}$ and $\Phi_{X}^{\mathrm{UD}}$ are defined by the following relationships:

$$
\begin{aligned}
\Phi_{X}^{D} & =\frac{\max \left(X^{D}, t=0 \cdots T\right)}{X_{\mathrm{ST}}^{D}}, \\
\Phi_{X}^{\mathrm{UD}} & =\frac{\max \left(X^{\mathrm{UD}}, t=0 \cdots T\right)}{X_{\mathrm{ST}}^{\mathrm{UD}}},
\end{aligned}
$$

where $T$ is the observation period and the subscript $(\cdot)_{\mathrm{ST}}$ refers to the value of the variable determined in a static analysis. Moreover, an additional description of the DAF is proposed to quantify the relationship between damaged and undamaged configurations by means of the following expression:

$$
\Phi_{X}^{D-\mathrm{UD}}=\frac{\max \left(X^{D}, t=0 \cdots T\right)}{X_{\mathrm{ST}}^{\mathrm{UD}}} .
$$

It is worth noting that the formulation of the DAF defined by (30) characterizes the dynamical amplification effects of the investigated variable with respect to the static response in the undamaged structural configuration of the bridge. This parameter can be useful for design purpose, since when this kind of DAF is known in advance the designer is able to control the amplification effects of a generic bridge variable due to the combined action produced by the failure mechanism and the inertial forces, avoiding the analysis suggested by the existing codes on the argument $[15,16]$. The bridge and moving load dimensioning is selected in accordance with the values utilized in practical applications and due, mainly, to both structural and economical reasons. The parametric study is developed by using dimensionless parameters, which are typically utilized to identify the structural and mechanical properties of a long-span bridge and moving loads. In particular, the cross-sectional stay areas are designed in such a way that the tensile stresses in the cables are always less than a design maximum allowable value, namely, $\sigma_{a}$, adopted on the basis of fatigue requirements. Such value is defined by the existing codes and it is considered as a known quantity, depending on the cross-section configuration and material technology $[9,10]$, which is, typically, provided by the manufactures of the cables and assumed as a fraction of the rupture stress of the steel [29]. At first, the stay dimensioning procedure is defined on the basis of the selfweight configuration. The stresses of a generic stay or the anchor stays are assumed to be equal to fixed working stress values, namely, $\sigma_{g}$ and $\sigma_{g 0}$, respectively, which are defined in the basis of the ratio between live and self-weight loads, the allowable stay stress, and the geometric characteristics of the bridges, by means of the following relationships [7, 10, 29-31]:

$$
\sigma_{g}=\frac{g}{g+p} \sigma_{a} \quad \sigma_{g 0}=\sigma_{a}\left\{1+\frac{p}{g}\left[1-\left(\frac{2 L}{l}\right)^{2}\right]^{-1}\right\}^{-1} .
$$

It is worth noting that since the stress increments in the stays can be supposed to be proportional to the live loads $p, \sigma_{g}$ and $\sigma_{g 0}$, defined on the basis of (28), are able to control the stress variation in the cable system produced by the live load application, avoiding that internal stresses in the stays exceed the allowable stress $\sigma_{a}$. Moreover, since the present analysis is devoted to investigate the behavior of long span bridges, it is reasonable to consider the girder as practically free from bending moments for reduced values of the stay spacing step and thus that it is dominated by means of a prevailing truss behavior $[9,10,29,30]$. As a consequence, it is possible to regard the deck as a continuous structure supported by a continuous distribution of stays, whose reaction forces can be calculated by using simple equilibrium relationships, able to provide cable dimensioning. In particular, the cross-sectional of the $i$ th stay area, namely, $A_{i}^{C}$, is designed in such a way that the dead loads produce a constant stress level over all the distributed elements equal to $\sigma_{g}$. Similarly, for the anchor stays the cross-sectional geometric area $A_{0}^{C}$ is designed in such a way that the allowable stress $\sigma_{g 0}$ is obtained for live loads $p$ applied to the central span only. Therefore, the geometric measurement for the cables system can be expressed by the following equations $[7-10,30]$ :

$$
\begin{gathered}
A_{i}^{C}=\frac{g \Delta_{i}}{\sigma_{g} \sin \alpha_{i}}, \\
A_{0}^{C}=\frac{g l}{2 \sigma_{g 0}}\left[1+\left(\frac{l}{H}\right)^{2}\right]^{1 / 2}\left[\left(\frac{L}{2 l}\right)^{2}-1\right],
\end{gathered}
$$


where $\alpha_{i}$ is the slope of a generic stay element with respect to the reference system, $(L, l, H)$ are representative geometric lengths of the bridge structure, and $\Delta$ is the stay spacing step. Moreover, aspect ratio, pylon stiffness, allowable cable stress and bridge, and moving loads characteristics are assumed as equal to the following representative values [7-10]:

$$
\begin{gathered}
\frac{L}{2 H}=2.5, \quad \frac{\ell}{H}=\frac{5}{3}, \quad \frac{L}{\Delta}=\frac{1}{100}, \\
a=\left(\frac{\gamma^{2} H^{2} E^{C}}{12 \sigma_{g}^{3}}\right), \quad \text { (bridge geometry) } \\
\frac{I_{2}^{G}}{I_{3}^{G}}=\frac{1}{10}, \quad \frac{I_{02}^{G}}{I_{03}^{G}}=\frac{1}{10}, \quad \frac{J_{t}^{G}}{A^{2}}=100, \\
\frac{I_{2}^{P}}{I_{3}^{P}}=\frac{1}{10}, \quad \frac{E^{G, P}}{C^{C}}=1 \quad(\text { girder and pylon }) \\
\varepsilon_{F}=\left(\frac{4 I_{2}^{G} \sigma_{g}}{H^{3} g}\right)^{1 / 4}, \quad \frac{K_{P}}{g}=50, \quad(\text { girder and pylon) } \\
\frac{\lambda_{\mathrm{ML}}^{0}}{\mu_{G} b^{2}}=1, \quad \frac{\lambda_{\mathrm{ML}}}{\mu_{G}}=1, \quad \frac{p}{g}=1, \\
\frac{e}{b}=0.5, \quad(\text { moving loads }) \\
\frac{A_{0}^{C} E^{C}}{g L}=100, \quad \tau_{0}=\frac{c t_{0}}{L}, \quad m=1, \\
\frac{\sigma_{a}}{C^{C}}=\frac{7.2 \mathrm{E} 8}{2.1 \mathrm{E} 11} \quad(\text { cable system }),
\end{gathered}
$$

where $H$ is the pylon height, $C^{C}$ is the modulus of elasticity of the cable, $K_{P}$ is the in-plane flexural top pylon stiffness, $b$ is half girder cross-section width, $t_{0}$ is the initial time in which the damage mechanism starts the degradation effects, $\tau_{0}$ is the normalized failure time, and $m$ is the parameter which controls the time evolution of the damage curve.

At first, the failure condition, located at the girder/cable intersection, is supposed to be produced in one anchor stay, located laterally to the longitudinal axis of the girder. The time of the failure mode is assumed to be consistent with values typically observed in experimental tests, whose representative value in the computations is assumed to be $0.005 \mathrm{sec}$ [16]. It is worth noting that additional analyses, not reported for the sake of brevity, show that the influence of the failure time step on the dynamical amplification factors is practically negligible and within $8 \%$ up to very high moving load speeds, that is, $c=160 \mathrm{~m} / \mathrm{s}$. Moreover, in this preliminary analysis it is assumed that the failure of the stay starts when the moving load front reaches the midspan, that is, $t_{R}=c /(l+L / 2)$. This configuration can be considered as an average value with respect to the position which assumes the moving system on the bridge development and will be taken as a reference in the subsequent developments, in

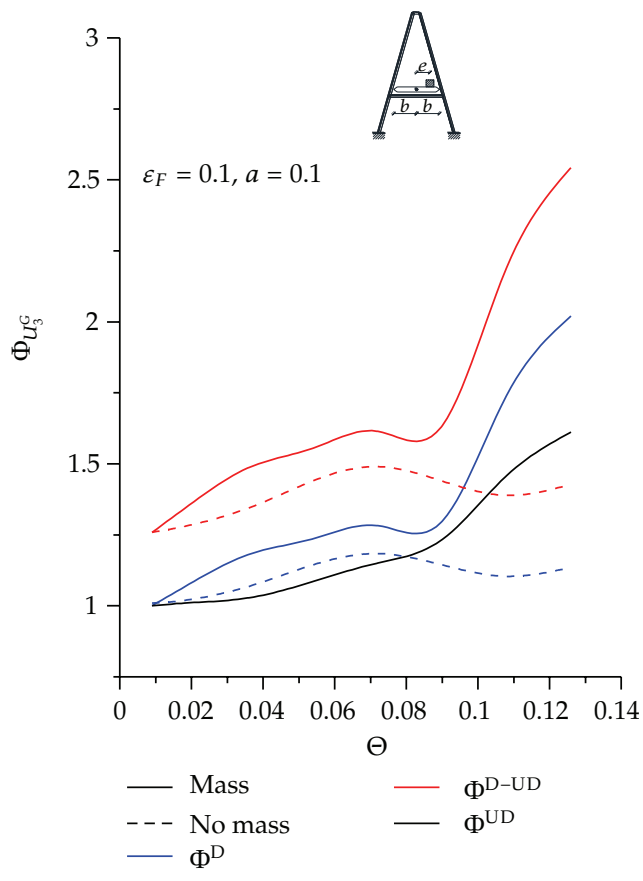

FIgURE 5: Dynamic amplification factors of the midspan vertical displacement for a bridge structure with A-shaped tower as a function of the normalized speed parameter: effect of the failure mechanism and moving load schematization.

which different instants where failure starts are considered. The behavior of the bridge is analyzed to investigate the relationship between dynamic amplification factors (DAFs) and the normalized speed parameter of the moving system, that is, $\Theta=c\left(\mu_{G} \sigma_{g} / E^{G} g H\right)^{1 / 2}$ as a function of the tower topology and the moving mass schematization. Moreover, the dynamic response of the bridge is evaluated by means of comparisons between damaged $(D)$ or undamaged bridge (UD) structures. The results, reported in Figures 5, 6, 7, and 8 , are defined through the relationships between moving system normalized speed and dynamic amplification factors for the midspan vertical displacement and bending moment. Nevertheless, the DAF evolution curves denote a tendency to increase with the speeds of the moving system. The results show that the DAFs developed for bridge structures affected by a failure mechanisms in the cable system are, typically, larger than those obtained assuming undamaged bridge configurations. Moreover, underestimations in the DAF predictions are observed in those cases, in which the inertial contributions arising from the external moving mass are completely neglected. The analyses presented above in terms of the DAFs $\Phi_{X}^{D-U D}$ for both the damaged and undamaged configurations point out that bridge structures with $\mathrm{A}$ - or $\mathrm{H}$-shaped typologies undergoing damage are characterized by large dynamic amplifications with respect to the undamaged case. As a matter of fact, the ranges of maximum value of the DAFs increase from [1.47-1.52] in the undamaged configuration to [2.5-3.6] in the damaged one for the midspan displacement, and similarly from [2.5-4.5] to [5.4-8.3] for the midspan bending moment. It is worth 


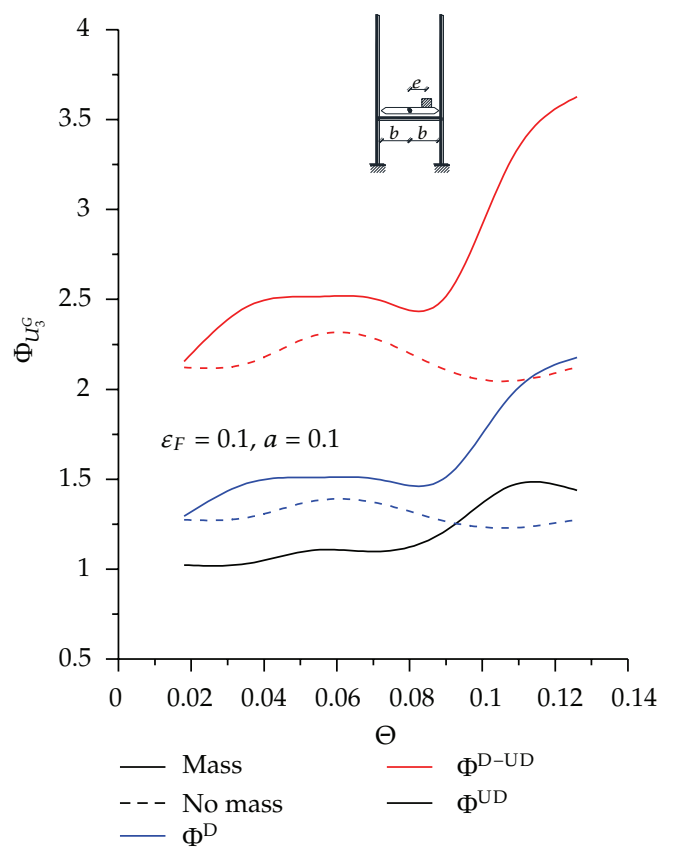

FIGURE 6: Dynamic amplification factors of the midspan vertical displacement for a bridge structure with $\mathrm{H}$-shaped tower as a function of the normalized speed parameter: effect of the failure mechanism and moving load schematization.

noting that the DAFs from the undamaged bridge configuration are affected by large amplifications, especially for the variables concerning the bending moments. This behavior can be explained in view of the prevailing truss behavior of the structure and the nonstandard inertial forces arising from the moving load application, which produce larger bending moments with respect to the ones obtained in the static configuration [8]. For all investigated cases, the bridge structures based on $\mathrm{H}$-shaped tower topology are affected by larger dynamic amplifications than those structures based on A-shaped tower. This behavior can be explained in view of the differences in the cable stress distribution between undamaged and damages structures. In particular, the $\mathrm{H}$ shaped tower bridges with respect to the A-shaped ones, owing to the failure of the lateral anchor stay, are affected by an unbalanced distribution of the internal stresses in the cable system, which produce larger torsional rotations and vertical displacements of the tower and the girder, respectively. To this aim, in Figure 9(a), a comparison of A- and Hshaped towers in terms of the DAFs $\left(\Phi_{\Phi_{1}^{G}}\right)$ and maximum observed value of the torsional rotation $\left(\bar{\Phi}_{1}\right)$ at the midspan cross-section is reported. These results show how the $\mathrm{H}$-shaped towers are much more affected by the investigated failure condition than the A-shaped towers, since lager torsional rotations of the tower and the girder are expected. In Figure 9(b), a synoptic representation of this deformation scheme affecting $\mathrm{H}$-shaped tower bridges is reported. Finally, the influence of the failure mode characteristics on the DAFs concerning the position which the moving system assumes on the bridge development is investigated. In particular, for a fixed value of the moving system speed, that is, $\vartheta=0.102$,

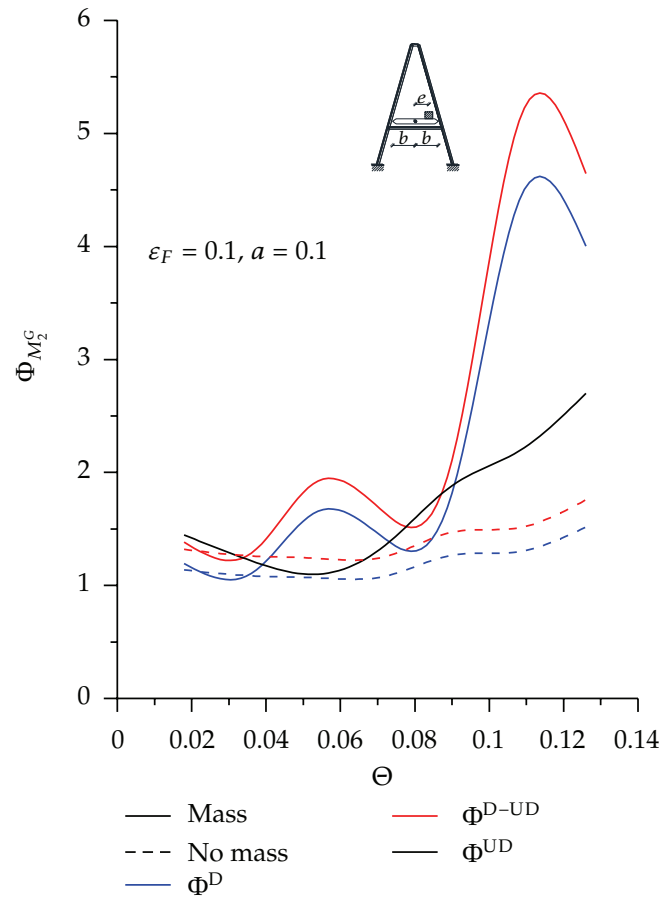

FIGURE 7: Dynamic amplification factors of the midspan bending moment for a bridge structure with A-shaped tower as a function of the normalized speed parameter: effect of the failure mechanism and moving load schematization.

analyses are developed in terms of normalized failure time $\tau_{0}$. The analyzed cases correspond to failure modes in which the moving load front is located at the entrance, the exit configurations, or at specified positions on the bridge development, that is, $X_{1} / L=[l / L,(l+L / 2) / L,(l+L) / L,(2 l+$ $L) / L]$. The results, reported in Tables 1 and 2 in terms of DAFs of the midspan vertical displacement and bending moment, show how the dynamic behavior is quite influenced by $\tau_{0}$ variable, since from a numerical point of view the values of the DAFs change, significantly, in the investigated ranges. Moreover, the results show that DAFs for damaged structures, much more for $\mathrm{H}$-shaped tower bridges, are typically larger than those evaluated for undamaged bridge cases.

Additional results are developed to investigate the effects of the bridge geometry on the DAFs and on the maximum values of typical bridge design variables for both damaged and undamaged bridge configurations. In particular, results are proposed in terms of the dimensionless parameter $a$, which describes the bridge size characteristics of the structure, and refer to a failure mechanism involving the complete failure of one lateral anchor stay of the cable system. The analyzed structures are consistent with a long-span bridge geometry, whose main span length varies from 500 to $1300 \mathrm{~m}$ and thus with a total length of the bridge between $900 \mathrm{~m}$ and $2100 \mathrm{~m}$. The results, reported in Figures 10, 11, 12, 13, 14 , and 15 concerning the undamaged configurations, show a tendency to decrease with increasing values of the bridge size variable. Contrarily, for damaged structures, the DAFs 


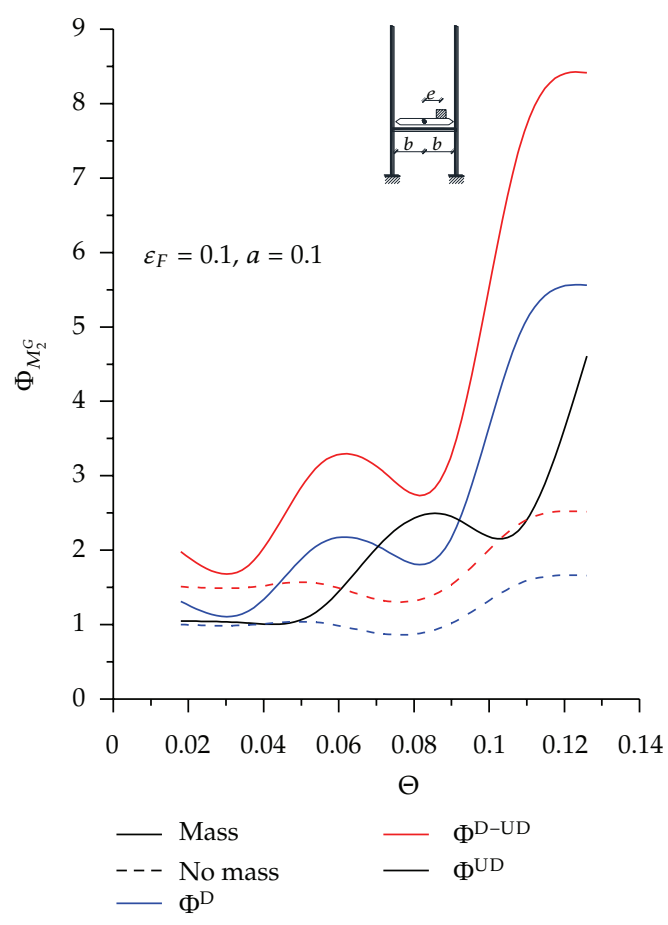

FIgURE 8: Dynamic amplification factors of the midspan bending moment for a bridge structure with $\mathrm{H}$-shaped tower as a function of the normalized speed parameter: effect of the failure mechanism and moving load schematization.

as well as the maximum values of the investigated kinematic and stress parameters display an oscillating behavior and some local peaks in curve development. The comparisons between damaged or undamaged bridge structures in terms of the tower typology show, essentially, that H-shaped tower structures are much more affected by damage failure. As a matter of fact, the DAFs defined by the ratios between dynamic damage value and the corresponding undamaged static quantity, that is, $\Phi_{X}^{D-\mathrm{UD}}$, show that, for the analyzed cases regarding $\mathrm{H}$-shaped tower typology, the displacements or the bending moments are typically greater than the bridge structures based on A-shaped tower typology. Similar conclusions can be drawn in terms of the maximum values of the observed variables. In particular, the comparisons between damaged and undamaged bridge behavior indicate that the A-shaped tower is able to reduce the increments produced by the failure mechanism, since the ratios defined in terms of displacements or bending moments are equal to 1.57 and to 1.66 , respectively. Contrarily, the results concerning $\mathrm{H}$-shaped tower denote that the undamaged variables are amplified with respect to factors equal to 2.2 and 2.5 for displacement and bending moments, respectively. A different behavior is observed with regard to the axial stresses of the undamaged anchor stay. In particular, the results reported in Figures 14 and 15 show that the $\mathrm{H}$-shaped tower bridges are affected by higher DAFs but lower maximum stresses with respect to the bridge structures based on A-shaped towers.

Finally, the behavior of the cable-stayed bridges is analyzed in terms of the failure mode, which affects the bridge structure. In particular, for both $\mathrm{H}$ - or A-shaped tower typologies, different damage mechanisms on the cable system are supposed on the cable system and the effects on the bridge structures are examined. The failure conditions, reported in Figure 16, are supposed to produce the complete collapse of the lateral anchor stay (Mode A) or the last three stays on both sides of the central part of the main span (Mode B). Additional modes of failure, concerning the damage to a number of internal stays of the cable system, are also investigated. However, since they do not produce any relevant effect on the investigated variables, for the sake of brevity, they are not discussed. The analyses are developed involving a typical geometry of the bridge structure, which is consistent with a long-span bridge-based typology. The results, reported in Figures 17, 18, 19, 20, 21, and 22, are proposed by means of comparisons of damaged and undamaged behavior, in terms of time histories and DAFs of the following cinematic and stress quantities:

(i) midspan displacements along $X_{3}$ axis of the girder, midspan torsional rotation of the girder;

(ii) bending moment in the $X_{1} X_{3}$ plane of the girder and undamaged anchor stay axial stress.

The analyses are developed for a fixed transit speed of the moving system, whereas the failure mechanism is supposed to begin when the moving system front reaches the midspan of the bridge, that is, $\Theta=0.102$ and $\tau_{0}=0.87$, respectively. The results show that the larger amplification effects on the vertical midspan displacement are obtained for the case in which the mode of failure affects the lateral anchor stay of the cable system, that is, Mode A. In this context, the vertical displacements from the undamaged bridge configuration are significantly amplified with respect to a multiplicative factor equal to 1.48 or to 2.28 for the A- or H-shaped tower topologies, respectively. Moreover, the DAFs for the investigated configurations are equal to 1.45 or 1.94 , whereas the DAFs defined as the ratio between $D$ on the UD quantities, that is, $\Phi_{U_{3}^{G}}^{D-\mathrm{UD}}$, are equal to 2.1 or to 3.2, respectively. The results concerning the bending moments indicate that the greater effects are produced by the damage mechanism affecting the central part of the cable system, that is, Mode B. In this context, the increments of the bending moments with respect to the undamaged values are equal to 3.2 or 4.1 for bridge configurations based on $\mathrm{H}$ - or A-shaped tower topology, respectively. Moreover, the maximum DAFs are equal to 4.63 and 8.19 for A- and $\mathrm{H}$-shaped tower typologies, respectively, and both of them refer to a damage condition involving the failure of the anchor stay. It is worth noting that the results concerning the cases of undamaged bridge structures based on A- or $\mathrm{H}$-shaped towers denote, essentially, the same prediction on the investigated variables. Contrarily, when damage mechanisms affect the cable system, notable amplifications of the investigated parameters are observed. In particular, the comparisons developed in terms of tower typologies indicate that $\mathrm{H}$ tower-based structures are much more affected by the damage mechanisms of the cable system than the A-shaped ones. This behavior can be explained by the fact that the failure of the anchor stay (Mode A) or 


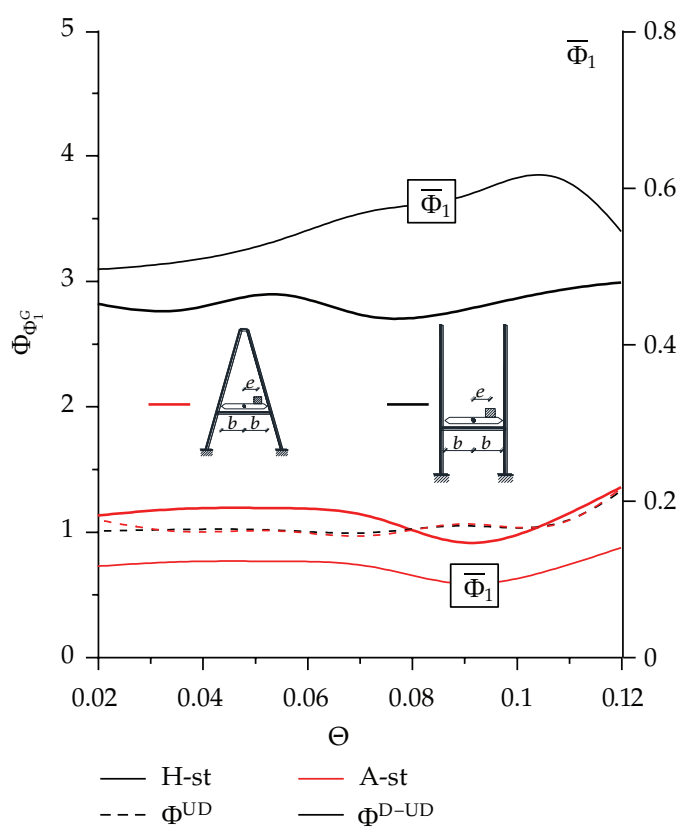

(a)

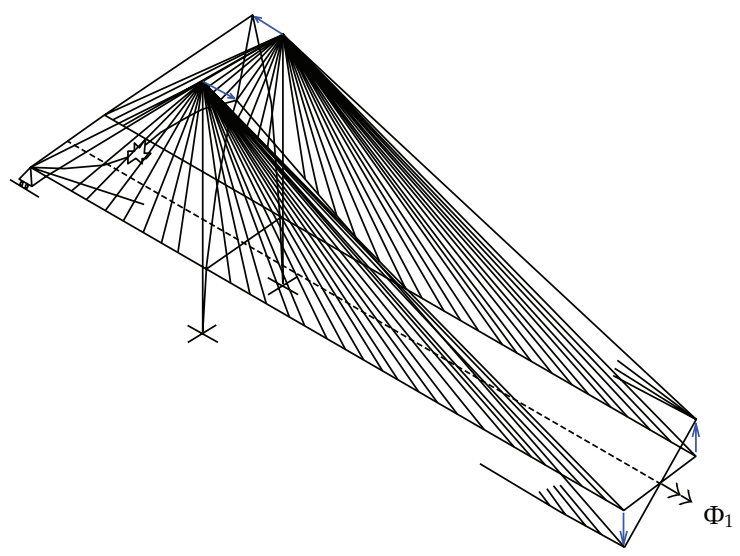

(b)

FIGURE 9: (a) Dynamic amplification factors and maximum value of the midspan torsional rotation as a function of the normalized speed parameter $\Theta$. (b) Schematic deformation produced by the failure mechanism.

TABLE 1: DAFs of the midspan bending moments as a function of the normalized time of failure on the bridge development comparisons in terms of $\mathrm{A}$ and $\mathrm{H}$ shaped.

\begin{tabular}{|c|c|c|c|c|c|c|}
\hline \multirow{2}{*}{$\tau_{0}$} & \multicolumn{3}{|c|}{$\mathrm{H}$-shaped tower } & \multicolumn{3}{|c|}{ A-shaped tower } \\
\hline & $\Phi^{D}$ & $\Phi^{D-\mathrm{UD}}$ & $\Phi^{\mathrm{UD}}$ & $\Phi^{D}$ & $\Phi^{D-U D}$ & $\Phi^{\mathrm{UD}}$ \\
\hline 0.00 & 6.26 & 9.48 & 2.81 & 5.85 & 6.84 & 3.24 \\
\hline 0.50 & 5.89 & 8.92 & 2.81 & 5.49 & 6.42 & 3.24 \\
\hline 0.87 & 7.52 & 11.39 & 2.81 & 6.10 & 7.13 & 3.24 \\
\hline 1.25 & 6.45 & 9.77 & 2.81 & 6.20 & 7.25 & 3.24 \\
\hline 1.62 & 4.82 & 7.30 & 2.81 & 5.84 & 6.82 & 3.24 \\
\hline 2.00 & 5.67 & 8.58 & 2.81 & 5.33 & 6.23 & 3.24 \\
\hline 2.50 & 1.38 & 2.09 & 2.81 & 3.26 & 3.81 & 3.24 \\
\hline
\end{tabular}

TABLE 2: DAFs of the midspan vertical displacements as a function of the normalized time of failure on the bridge development comparisons in terms of $\mathrm{A}$ and $\mathrm{H}$ shaped.

\begin{tabular}{|c|c|c|c|c|c|c|}
\hline \multirow{2}{*}{$\tau_{0}$} & \multicolumn{3}{|c|}{ H-shaped tower } & \multicolumn{3}{|c|}{ A-shaped tower } \\
\hline & $\Phi^{D}$ & $\Phi^{D-\mathrm{UD}}$ & $\Phi^{\mathrm{UD}}$ & $\Phi^{D}$ & $\Phi^{D-U D}$ & $\Phi^{\mathrm{UD}}$ \\
\hline 0.00 & 1.973 & 3.046 & 1.451 & 1.62 & 2.03 & 1.60 \\
\hline 0.50 & 1.890 & 2.918 & 1.451 & 1.58 & 1.98 & 1.60 \\
\hline 0.87 & 2.032 & 3.137 & 1.451 & 1.75 & 2.20 & 1.60 \\
\hline 1.25 & 2.096 & 3.237 & 1.451 & 1.72 & 2.15 & 1.60 \\
\hline 1.62 & 2.264 & 3.495 & 1.451 & 1.61 & 2.02 & 1.60 \\
\hline 2.00 & 2.238 & 3.456 & 1.451 & 1.65 & 2.07 & 1.60 \\
\hline 2.50 & 0.940 & 1.451 & 1.451 & 1.26 & 1.59 & 1.60 \\
\hline
\end{tabular}

the stays in the central part (Mode B) produce unbalanced forces in the cable system and thus on the girder leading to high rotations of the pylons and the girder along vertical $X_{3}$ and $X_{3}$ axes, respectively. This behavior is confirmed by the results reported in Figures 21 and 22, in which time histories of the torsional rotation and anchor stay axial force in terms of the damage mechanism and tower typology are reported. The analyses show that bridge structures based 


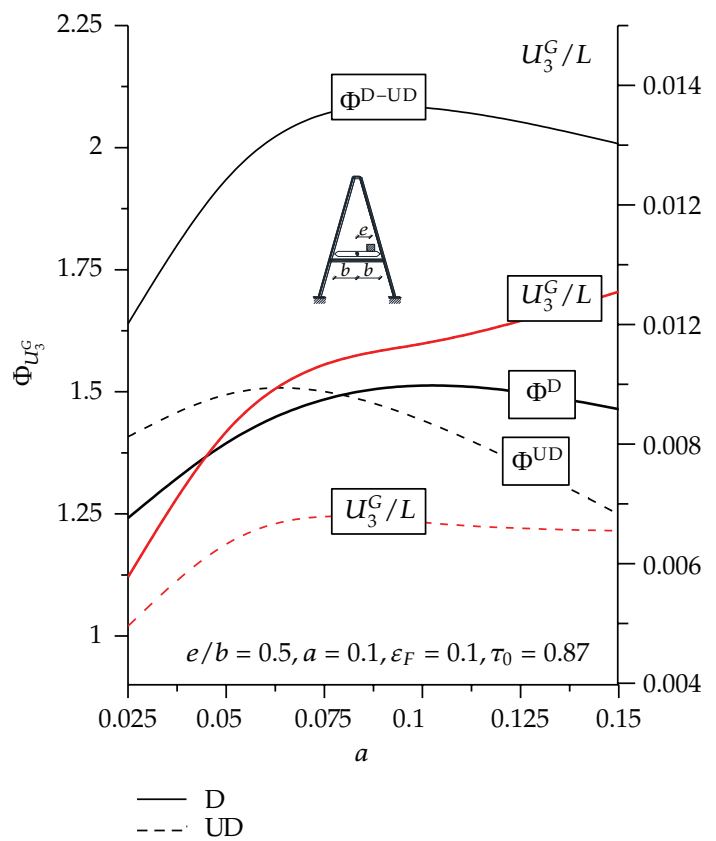

Figure 10: Dynamic amplification factors of the midspan vertical displacement for a bridge structure with A-shaped tower: response of damaged and undamaged bridge structures as a function of the bridge size parameter $a$.

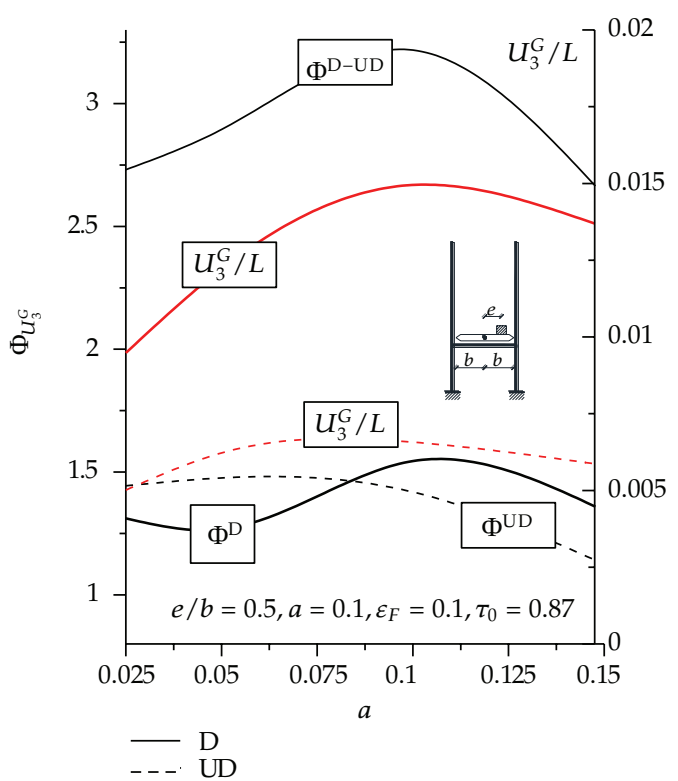

FIGURE 11: Dynamic amplification factors of the midspan vertical displacement for a bridge structure with $\mathrm{H}$-shaped tower: response of damaged and undamaged bridge structures as a function of the bridge size parameter $a$.

on A-shaped tower are practically unaffected by the failure modes, since the same prediction on the maximum torsional rotations is observed. On the contrary, the $\mathrm{H}$-shaped tower bridges owing to the concurrent rotations of the girder and the pylons are affected by greater deformations and DAFs. Moreover, the results shown in terms of the anchor stay axial

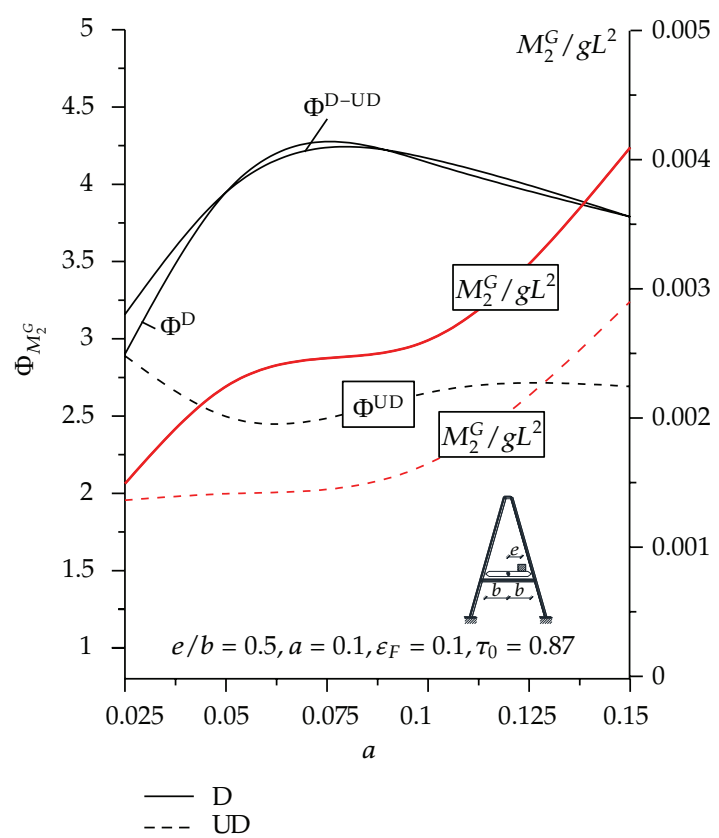

Figure 12: Dynamic amplification factors of the midspan bending moment for a bridge structure with A-shaped tower: response of damaged and undamaged bridge structures as a function of the bridge size parameter $a$.

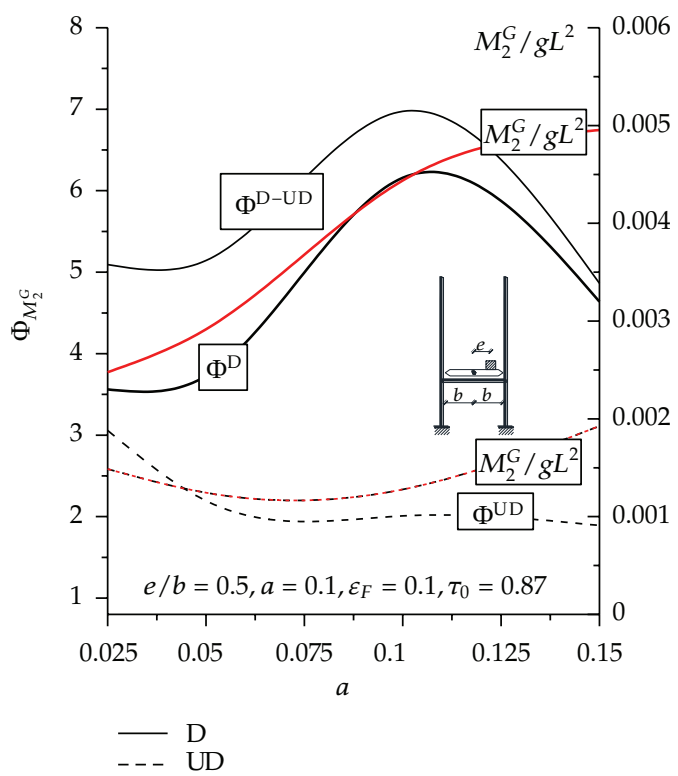

FIGURE 13: Dynamic amplification factors of the midspan bending moment for a bridge structure with $\mathrm{H}$-shaped tower: response of damaged and undamaged bridge structures as a function of the bridge size parameter $a$.

stresses, reported in Figure 22, denote that "Mode A" failure mechanism produces high increments in the axial stress, mainly, for the A-shaped tower typology. This behavior can be explained by the geometric configuration of the A-shaped pylon and by its ability to redistribute the internal stresses from the damaged to the undamaged anchor stay. 


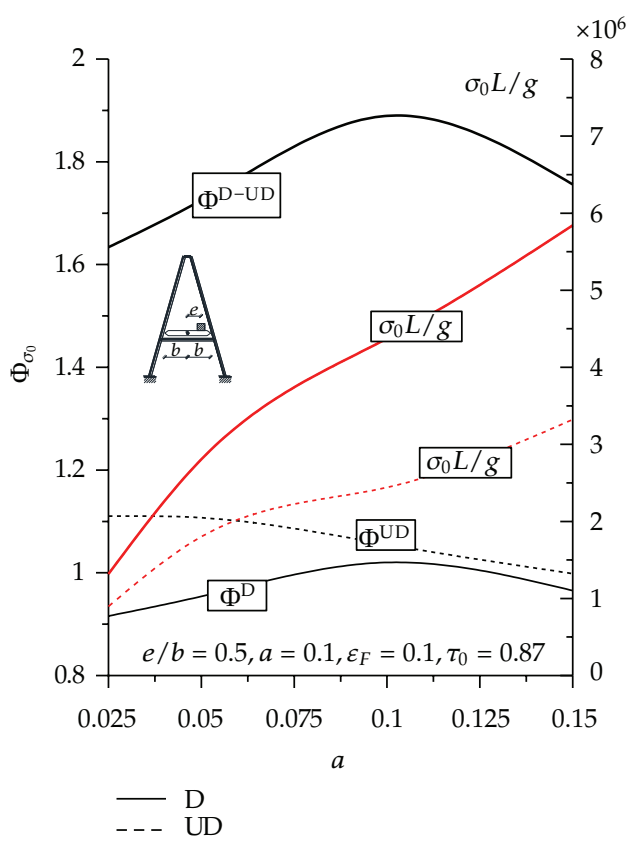

FIGURE 14: Dynamic amplification factors of the undamaged anchor stay axial stress for A-shaped tower bridge configurations: damaged and undamaged bridge response as a function of the bridge size parameter $a$.

\section{Conclusions}

Long-span bridges under moving loads have been analyzed by means of tridimensional deformation modes, in terms of dynamic impact factors and maximum values of typical design bridge variables. The proposed model takes into account nonlinearities involved by large displacements effects, whereas in those stays affected by internal damage, time dependent damage functions are introduced in the constitutive relationships. The purpose of this investigation is to analyze the amplification effects of the bridge structure produced by the moving load application and damage mechanisms in the cable system. The analyses have shown that the presence of damage mechanism in the cable system is able to produce larger DAFs then those obtained for undamaged bridge configurations. The DAFs strongly depend on the moving system speeds and the mass schematization. Underestimations in prediction of DAFs and maximum design bridge variables are noted for the cases in which the inertial description of the moving mass is not properly taken into account. The results developed in terms of the damage mechanism configuration, moving mass description, and bridge properties have shown that recommendations provided by existing codes, that is, PTI [15] and SETRA [16], become unsafe in many cases. As a matter of fact, results developed in terms of the damage mechanism characteristics have shown that the damage mode which produces the worse effects on the bridge behavior is that associated with the failure of the anchor stay. In particular, the analyses have pointed out that damage mechanisms involving the failure of the lateral anchor stay are able to produce large

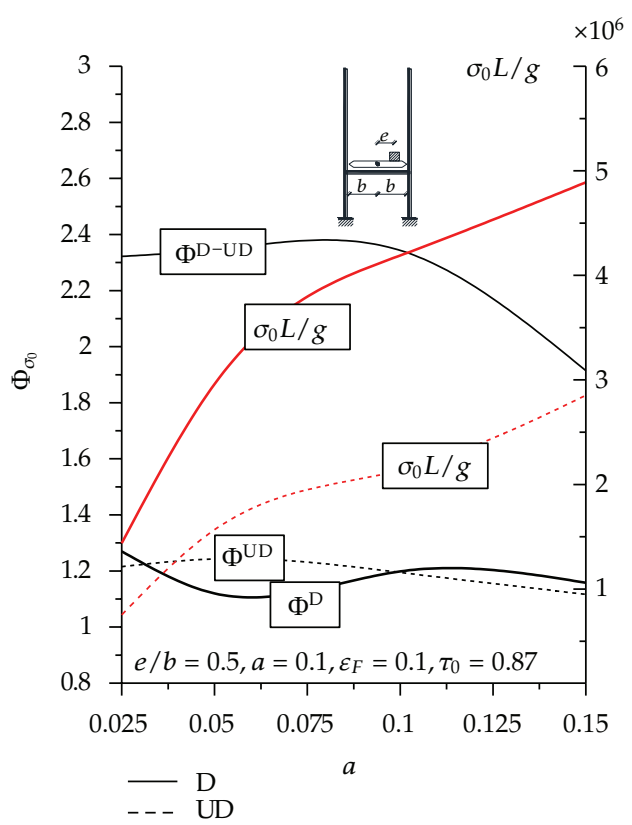

FIGURE 15: Dynamic amplification factors of the undamaged anchor stay axial stress for $\mathrm{H}$-shaped tower bridge configurations: damaged and undamaged bridge response as a function of the bridge size parameter $a$.

amplifications in the investigated parameters, whose ranges with respect to the static undamaged value are equal to 2.53.5 for vertical midspan displacement, to 5.5-8.5 for midspan bending moment, to 1.3-2.8 for midspan torsional rotation, and to 1.9-2.3 for the anchor stay axial stress. The results developed in terms of the damage mechanism characteristics have shown that the damage mode, which produces the worse effects on the bridge behavior is that associated with the failure of the anchor stay. Comparisons developed in terms of tower topology have shown that the $\mathrm{H}$-shaped tower bridge is much more affected than the A-shaped one, since the failure modes produce an unbalanced distribution of the internal stresses in the cable system, leading to larger torsional rotations and vertical displacements of the tower and the girder, respectively. It is worth noting that, in the present paper, only the effects produced by moving loads are considered in the results. However, in the framework of cable-stayed bridges, another severe loading condition is the one related to wind effects. As a matter of fact, damage mechanisms in the cable system, among which a typical example is the one considered in the present paper, may amplify the resonance effects related to aeroelastic instability phenomena, leading to a premature bridge collapse. However, the study of the coupled aeroelastic and damage effects is beyond the scope of the present paper and will be an object of future investigation.

\section{Appendix}

The damage description is developed by means of a phenomenological approach based on the Continuum Damage 


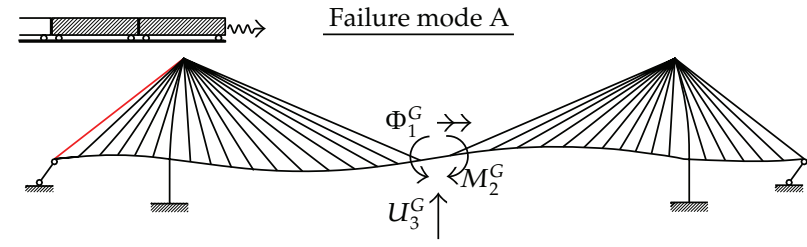

(a)

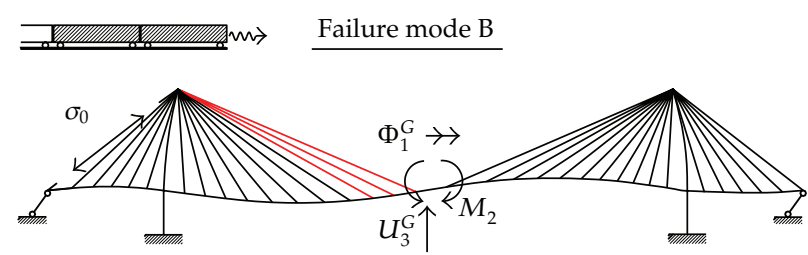

(b)

FIGURE 16: Synoptic representation of the failure modes: (a) failure in the lateral anchor stay and (b) failure in the central part of the cable system.

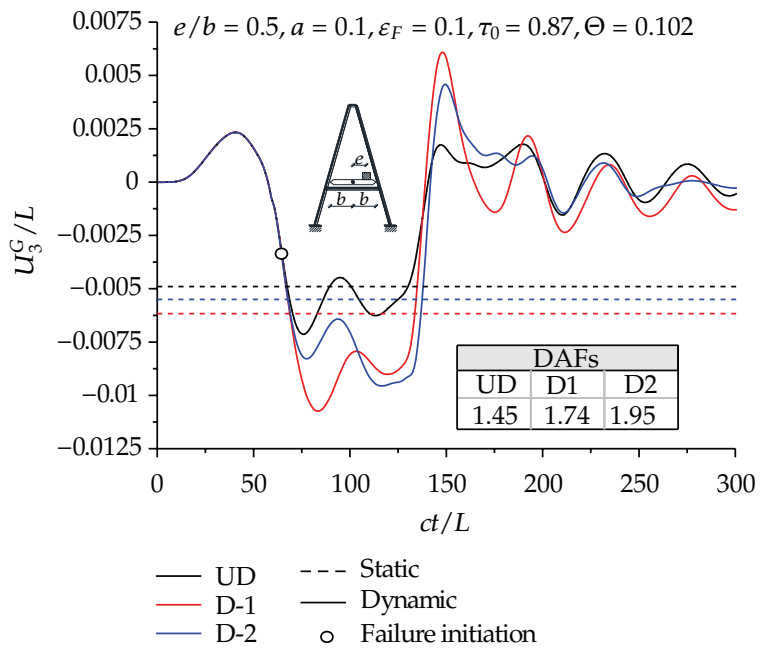

FIGURE 17: Time history of the dimensionless midspan displacement and evaluation of the DAFs: comparisons between damaged and undamaged A-shaped bridge configurations.

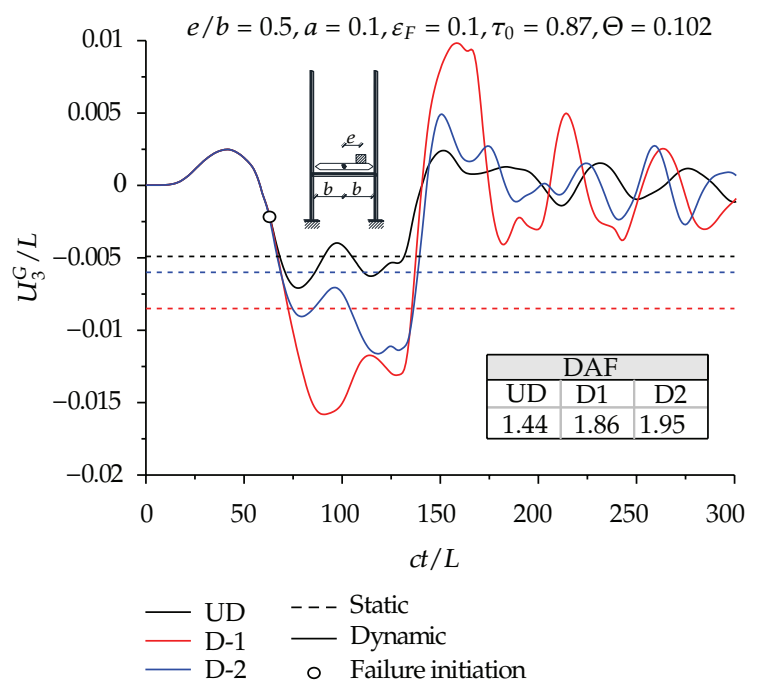

FIGURE 18: Time history of the dimensionless midspan displacement and evaluation of the DAFs: comparisons between damaged and undamaged $\mathrm{H}$-shaped bridge configurations.

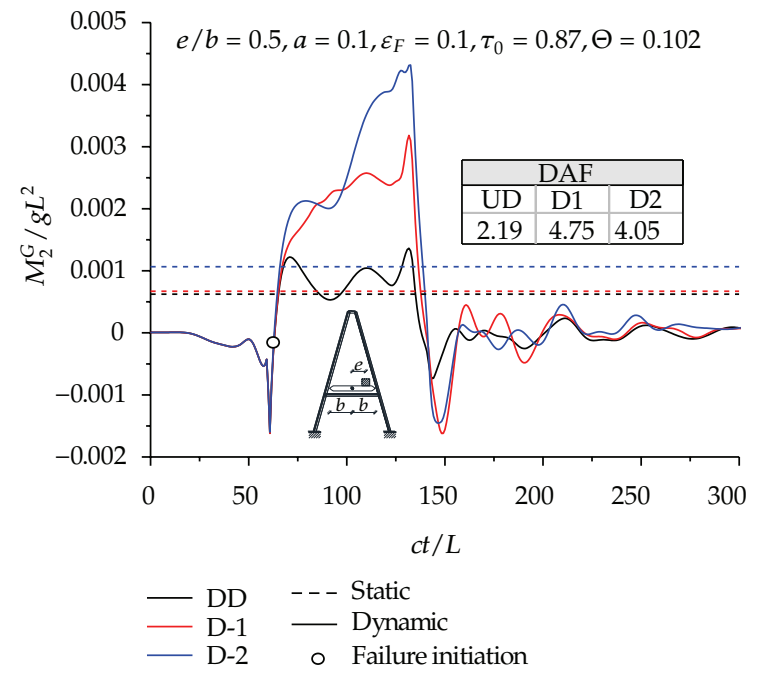

FIgURE 19: Time history of the dimensionless midspan bending moment and evaluation of the DAFs: comparisons between damaged and undamaged A-shaped bridge configurations.

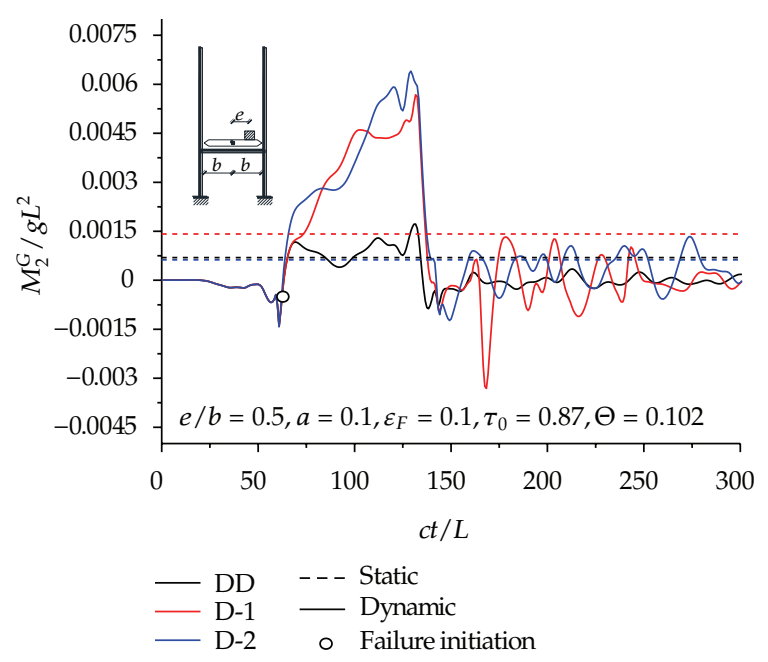

FIGURE 20: Time history of the dimensionless midspan bending moment and evaluation of the DAFs: comparisons between damaged and undamaged $\mathrm{H}$-shaped bridge configurations. 


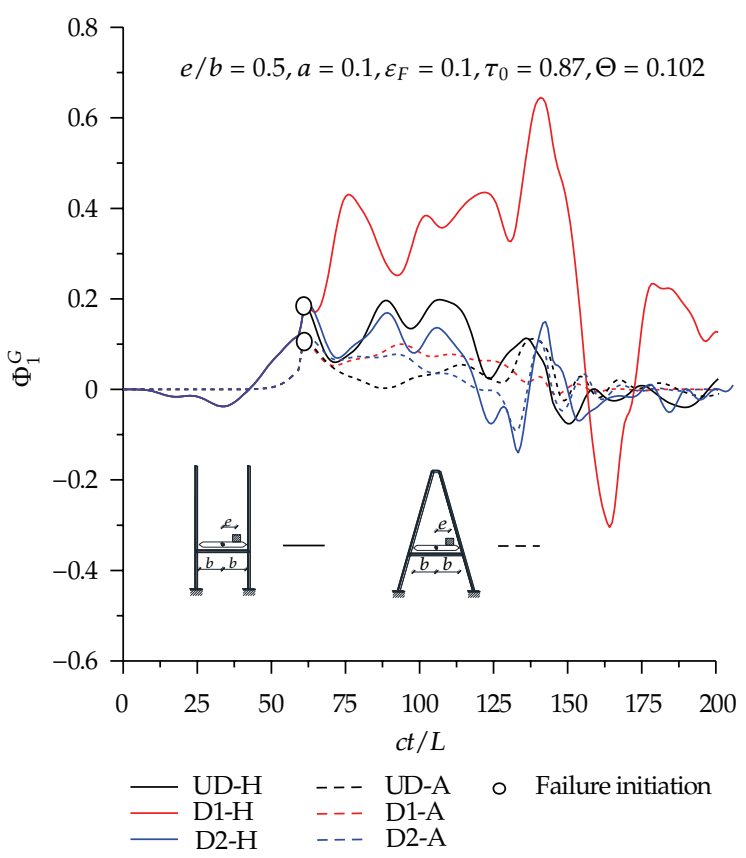

FIGURE 21: Time history of the dimensionless midspan torsional rotation: comparisons in terms of the failure mode and tower typology.

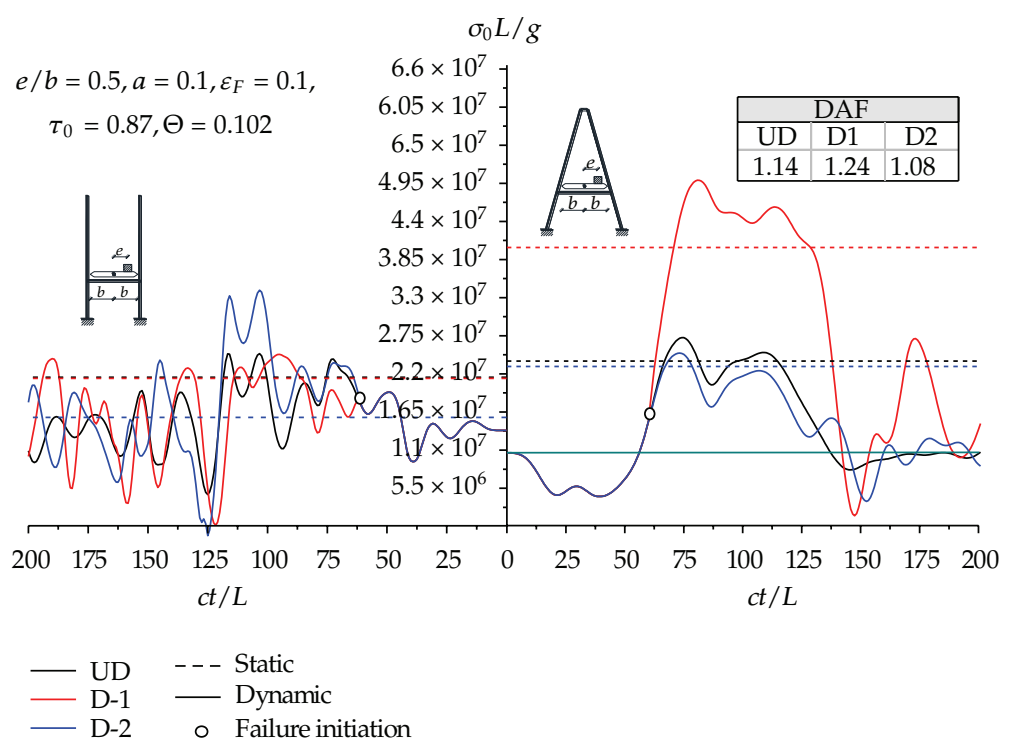

FIGURE 22: Time history of the dimensionless of the undamaged anchor stay axial force: comparisons in terms of the failure mode and tower typology.

Mechanics. The damage evolution is determined by means of a Kachanov type law based on the following expression [22]:

$$
\frac{d D(s, t)}{d t}=\left[\frac{S}{A_{0}(1-D(s, t))}\right]^{m}
$$

where $A_{0}$ and $m$ are two characteristic damage coefficients for the material. Introducing the initial condition corresponding to the undamaged state, that is, $D(0)=0$, and, integrating (A.1), the damage evolution law is obtained as

$$
D(s, t)=1-\left[-\frac{A_{0}^{m} S^{-m}}{(m+1) t-S^{-m} A_{0}^{m}}\right]^{1 /(m+1)} .
$$

The time to failure $\left(t_{f}\right)$ is obtained from (A.2) and enforcing the failure condition defined in terms of 
the critical damage value by means of the following relationships:

$$
D\left(s, t_{f}\right)=D_{c}, \quad t_{f}=\frac{1-\left(1-D_{c}\right)^{m+1}}{m+1}\left(\frac{S}{A_{0}}\right)^{-m} .
$$

Finally, substituting (A.3) into (A.2), the damage law can be expressed by the following relationship:

$$
D(s, t)=1-\left(1-\frac{t_{f}-\left(t-t_{0}\right)}{t_{f}}\right)^{1 /(1+m)},
$$

\section{Nomenclature}

$(\cdot)^{G}$ : Stiffening girderproperty

$(\cdot)^{P}$ : Pylon property

$(\cdot)^{C}$ : Cablesystemproperty

$(\cdot)^{m}$ : Movingmassproperty

$\alpha_{i}$ : Longitudinal geometric slope of $i$ th stay

$\alpha_{0}$ : Longitudinal anchor stay geometric slope

$A^{G}$ : Girder cross sectional area

$A^{C}$ : Stay cross sectional area of the $i$ th stay

$A_{0}^{C}$ : Anchor stay cross sectional area

$b$ : Half girder cross section width

$C^{C}$ : Stay elastic modulus

c: Moving system speed

$\Delta: \quad$ Stay spacing step

$D$ : Damage variable

$D_{c}$ : Critical value of the damage variable

$E^{G}$ : Elasticity modulus of the girder

$e$ : Eccentricity of the moving loads with respect to the girder geometric axis

$g$ : Girder self-weight per unit length

$\gamma$ : Stay specific weight

$H$ : Pylon height

$I_{i}$ : $\quad$ Moment of inertia with respect to the $i$-axis

$I_{0 i}$ : Polar moment of inertia around the $i$-axis

$J_{t}: \quad$ Factor torsional stiffness

$K_{P}$ : Flexural top pylon stiffness

$k$ : $\quad$ Undamaged stiffness of the stay

$k_{d}$ : Damaged stiffness of the stay

$\lambda_{\mathrm{ML}}$ : Moving load mass per unit length

$\lambda_{\mathrm{ML}}^{0}$ : Torsional polar mass moment of moving load per unit length

$L: \quad$ Central bridge span

$L_{p}$ : Length of the moving loads

$l$ : $\quad$ Lateral bridge span

$\mu_{C}$ : Stay mass per unit length

$\mu_{G}:$ Girder mass per unit length

p: $\quad$ Live loads

$U_{i}$ : Component of the displacement field along $i$ axis

$\Phi_{i}$ : Component of the rotation field around $i$ axis

$\sigma_{g}: \quad$ Design stay stress under self-weight loading

$\sigma_{a}:$ Allowable stay stress

$S_{i}$ : Stay stress in the "zero configuration"

$S_{0}$ : Initial trial stay stress

$\tau_{0}$ : Normalized failure time $t_{0}$ : Initial time of the failure mechanism

$t_{f}$ : Final time of the failure mechanism

$t_{R}$ : Duration of the failure mechanism

$X_{i}$ : Component of the positional vector of the cable cross section.

\section{References}

[1] Y. S. Cheng, F. T. K. Au, and Y. K. Cheung, "Vibration of railway bridges under a moving train by using bridge-track-vehicle element," Engineering Structures, vol. 23, no. 12, pp. 1597-1606, 2001.

[2] H. Zhang and X. Xie, "Dynamic responses of cable-stayed bridges to vehicular loading including the effects of the local vibration of cables," Journal of Zhejiang University, vol. 12, no. 8, pp. 593-604, 2011.

[3] F. Yang and G. A. Fonder, "Dynamic response of cable-stayed bridges under moving loads," Journal of Engineering Mechanics, vol. 124, no. 7, pp. 741-747, 1998.

[4] H. L. Wang, S. F. Qin, Z. Zhang, C. L. Huang, and W. J. Xu, "The Basic differential equations of self-anchored cable-stayed suspension bridge," Mathematical Problems in Engineering, vol. 2010, Article ID 805195, 2010.

[5] F. T. K. Au, J. J. Wang, and Y. K. Cheung, "Impact study of cable-stayed bridge under railway traffic using various models," Journal of Sound and Vibration, vol. 240, no. 3, pp. 447-465, 2001.

[6] F. T. K. Au, J. J. Wang, and Y. K. Cheung, "Impact study of cable-stayed railway bridges with random rail irregularities," Engineering Structures, vol. 24, no. 5, pp. 529-541, 2002.

[7] D. Bruno, F. Greco, and P. Lonetti, "Dynamic impact analysis of long span cable-stayed bridges under moving loads," Engineering Structures, vol. 30, no. 4, pp. 1160-1177, 2008.

[8] D. Bruno, F. Greco, and P. Lonetti, "A parametric study on the dynamic behavior of combined cable-stayed and suspension bridges under moving loads," International Journal for Computational Methods in Engineering Science and Mechanics, vol. 10, no. 4, pp. 243-258, 2009.

[9] M. S. Troitsky, Cable Stayed Bridges, Crosby Lockwood Staples, London, UK, 1977.

[10] N. J. Gimsing, Cable Supported Bridges: Concepts and Design, John Wiley \& Sons, New York, NY, USA, 1997.

[11] P. K. Chatterjee, T. K. Datta, and C. S. Surana, "Vibration of cable-stayed bridges under moving vehicles," Structural Engineering International, vol. 4, pp. 116-121, 1994.

[12] T. G. Konstantakopoulos and G. T. Michaltsos, "A mathematical model for a combined cable system of bridges," Engineering Structures, vol. 32, no. 9, pp. 2717-2728, 2010.

[13] M. Lepidi, V. Gattulli, and F. Vestroni, "Static and dynamic response of elastic suspended cables with damage," International Journal of Solids and Structures, vol. 44, no. 25-26, pp. 8194-8212, 2007.

[14] J. Zhu, G. R. Ye, Y. Q. Xiang, and W. Q. Chen, "Dynamic behavior of cable-stayed beam with localized damage," Journal of Vibration and Control, vol. 17, no. 7, pp. 1080-1089, 2011.

[15] Post-Tensioning Institute, Recommendations for Stay Cable Design, Testing and Installation, Post-Tensioning Institute, Phoenix, Ariz, USA, 2007.

[16] SETRA, Haubans-Recommandations de la Commission Interministérielle de la Précontrainte, Service d'Etudes Techniques des Routes et Autoroutes, Bagneux, France, 2001. 
[17] D. Bruno, F. Greco, P. Lonetti, and O. Nevone Blasi, "Dynamic interaction of cable supported bridges with traffic loads including the effect of an accidental failure in the cable system. bridge maintenance, safety, management, resilience and sustainability," in Proceedings of the 6th International Conference on Bridge Maintenance, Safety and Management, pp. 2827-2834, 2012.

[18] M. Wolff and U. Starossek, "Cable loss and progressive collapse in cable-stayed bridges," Bridge Structures, vol. 5, pp. 17-28, 2009.

[19] U. Starossek, "Avoiding disproportionate collapse of major bridges," Structural Engineering International, vol. 19, no. 3, pp. 289-297, 2009.

[20] P. Wriggers, Nonlinear Finite Element Methods, Springer, Berlin, Germany, 2008.

[21] J. Lemaitre and J. L. Chaboche, Mechanics of Solid Materials, Cambridge University Press, Cambridge, UK, 1994.

[22] E. J. Barbero, P. Lonetti, and K. K. Sikkil, "Finite element continuum damage modeling of plain weave reinforced composites," Composites B, vol. 37, no. 2-3, pp. 137-147, 2005.

[23] P. Warnitchai, Y. Fujino, and T. Susumpow, "A non-linear dynamic model for cables and its application to a cablestructure system," Journal of Sound and Vibration, vol. 187, no. 4, pp. 695-712, 1995.

[24] H. Xia, Y. L. Xu, and T. H. T. Chan, "Dynamic interaction of long suspension bridges with running trains," Journal of Sound and Vibration, vol. 237, no. 2, pp. 263-280, 2000.

[25] K. Kawashima, S. Unjoh, and M. Tunomoto, "Estimation of damping ratio of cable-stayed bridges for seismic design," Journal of Structural Engineering, vol. 119, no. 4, pp. 1015-1031, 1993.

[26] H. Yamaguchi and M. Ito, "Mode-dependence of structural damping in cable-stayed bridges," Journal of Wind Engineering and Industrial Aerodynamics, vol. 72, no. 1-3, pp. 289-300, 1997.

[27] L. Fryba, Vibration of Solids and Structures Under Moving Loads, Thomas Telford, London, UK, 1999.

[28] Comsol, Reference Manual. Stockholm: Comsol AB, 2010.

[29] B. Houriet, J. F. Klein, W. Isler, P. Moia, and R. Walther, Cable Stayed Bridges, Thomas Telford Publishing, 2nd edition, 2003.

[30] M. Como, A. Grimaldi, and F. Maceri, "Statical behaviour of long-span cable-stayed bridges," International Journal of Solids and Structures, vol. 21, no. 8, pp. 831-850, 1985.

[31] D. Bruno and A. Leonardi, "Natural periods of long-span cablestayed bridges," Journal of Bridge Engineering, vol. 2, no. 3, pp. 105-115, 1997. 


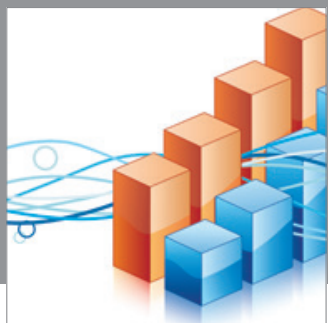

Advances in

Operations Research

mansans

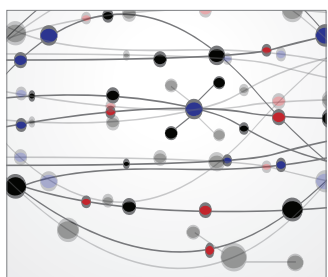

The Scientific World Journal
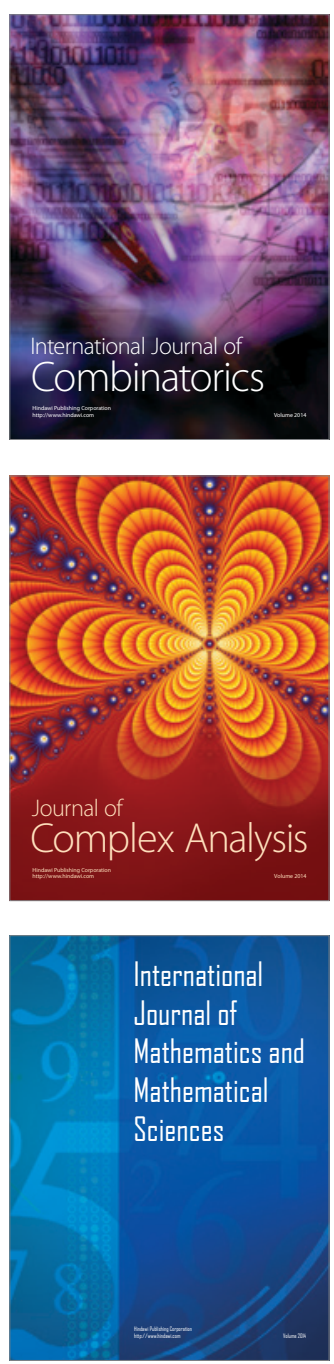
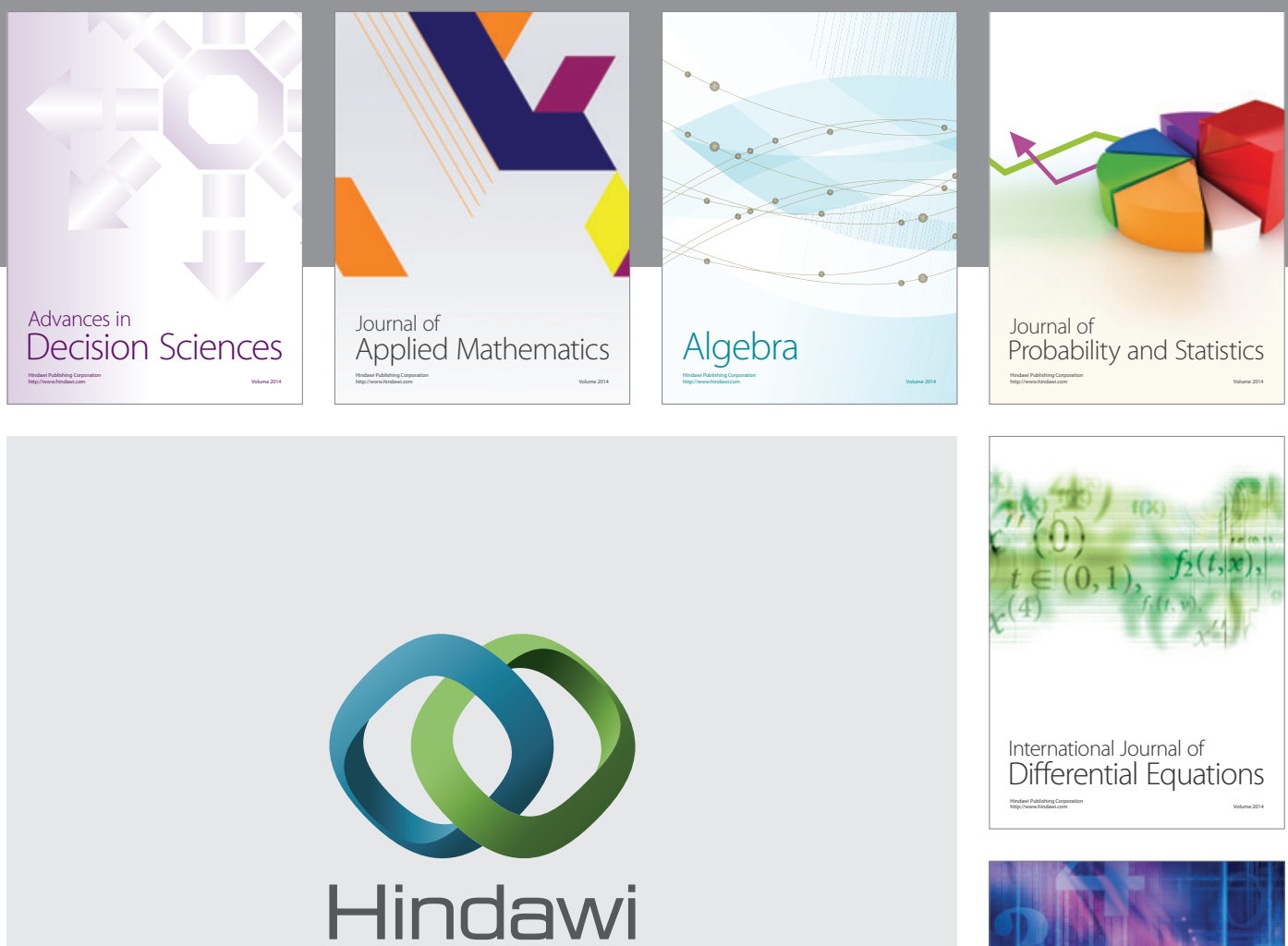

Submit your manuscripts at http://www.hindawi.com
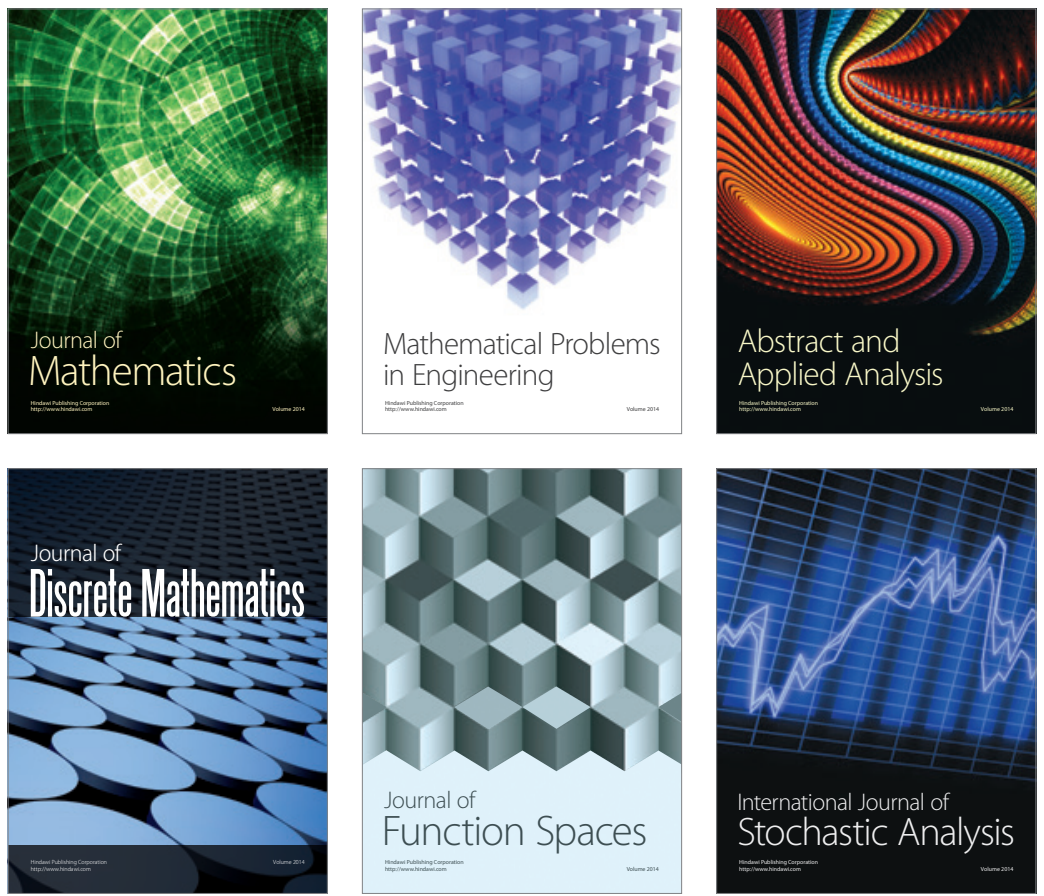

Journal of

Function Spaces

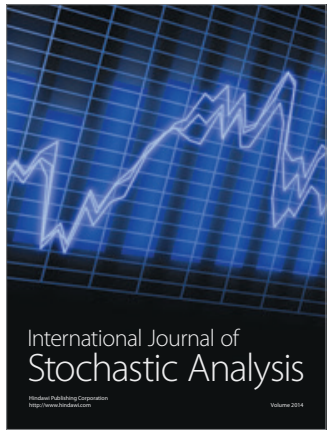

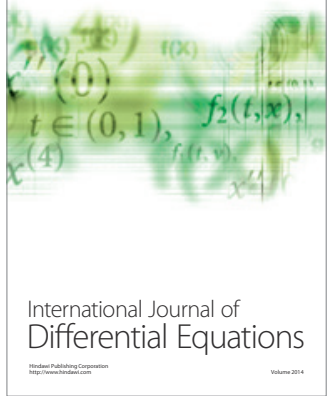
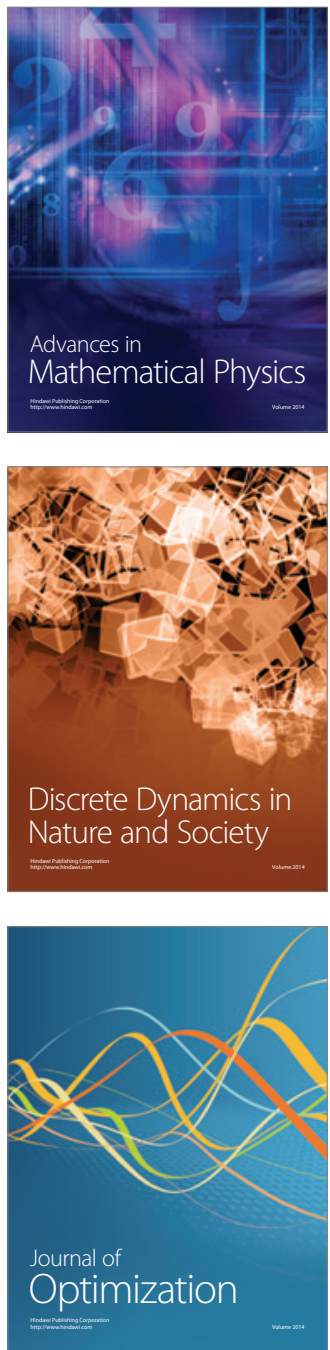